OPEN ACCESS

Edited by:

Andrea Valdés-Hernández, National Autonomous University of

Mexico, Mexico

Reviewed by:

Theo Nieuwenhuizen,

University of Amsterdam, Netherlands

Emmanuel E. Haven,

Memorial University, Canada

Robert Bruce Mann,

University of Waterloo, Canada

${ }^{*}$ Correspondence:

William Sulis

sulisw@mcmaster.ca

Specialty section:

This article was submitted to Mathematical and Statistical Physics,

a section of the journal

Frontiers in Physics

Received: 24 April 2020

Accepted: 28 July 2020

Published: 15 September 2020

Citation:

Sulis W (2020) Locality Is Dead! Long

Live Locality!. Front. Phys. 8:360.

doi: 10.3389/fphy.2020.00360

\section{Locality Is Dead! Long Live Locality!}

\author{
William Sulis*
}

Collective Intelligence Laboratory, Department of Psychiatry and Behavioural Neuroscience, McMaster University, Hamilton, ON, Canada

Several decades of theory and experiment into EPR correlations have led to the widely held belief that reality is non-local, in spite of the fact that this violates special relativity. To date, no experiment has shown a violation of special relativity, and EPR experiments do not demonstrate the existence of superluminal information exchange, merely correlations which violate certain inequalities. Every "loophole" in these hidden variable theories has been thought plugged. However, there is much confusion in the literature due to conflation of the terms "locality," "realism," "hidden variables," "non-contextuality." The presence of local hidden variables is thought to necessarily lead to a Kolmogorov probability structure (hence non-contextuality), but this is an assumption, one which is not true in general once context effects are taken into account. Treated as an observational theory, several authors have shown no incompatibility between quantum mechanics and locality, and that the Bell scenario is actually about whether reality is contextual. This paper proposes a descriptive theory by assuming a generated reality (following Whitehead's Process Theory) which can violate the principle of continuity and possess non-Kolmogorov probability structure, and reproduce the results of non-relativistic quantum mechanics, while allowing only causally local information exchange without hidden variables. A generated reality is thus compatible with both quantum mechanics and special relativity, reproducing all of the results expected from quantum mechanics while still maintaining causally local realism. This process model thus appears to be an ideal candidate for developing theories for the unification of quantum mechanics and general relativity.

Keywords: local realism, contextuality, process algebra, process model, non-relativistic quantum mechanics

\section{INTRODUCTION}

The debate as to whether reality at its fundamental level conforms to the tenets of local realism has been decided decisively in the negative. Non-locality has won. Locality is dead! Or is it? In spite of an ever more sophisticated series of hidden variable theorems and dramatic experimental results, has the issue of local realism truly been laid to rest? In the years following the seminal paper of Einstein, Podolsky and Rosen (EPR) [1], the concept of local realism has become equated with the concept of (deterministic) local, non-contextual, hidden variables (LNHV). The assumption of LNHV leads to inequalities on measurement correlations, which experiments have shown are violated. The conclusion is that LNHV do not exist.

The restriction of local hidden variables to deterministic LNHV was unnecessary, likewise, the restriction of local realism to LNHV is also excessive. Arguments in support of this will be provided and an explicit model of non-relativistic quantum mechanics (NRQM), the Process Algebra model, will be demonstrated. The Process Algebra model is a local, realist, generative, contextual, and 
discrete model of NRQM without hidden variables in which NRQM appears as an effective theory in the continuum limit as Planck length and time are taken to zero [2-6]. It has been argued that the Process Algebra model provides a true completion of NRQM [4]. The Process Algebra provides a nuanced framework for representing interactions between fundamental entities which the standard Hilbert space formulation lacks. It suggests that the quantum paradoxes and conundrums are due to a failure of the usual Hilbert space formalism to correctly represent particle interactions.

\section{THE ARGUMENTS AGAINST LOCAL HIDDEN VARIABLES}

The story against local realism begins with the 1935 EPR paper [1], which tackled two questions, that of the completeness of quantum mechanics as a physical theory and that of the nature of reality. As to completeness, they wrote: "Whatever the meaning assigned to the term complete, the following requirement for a complete theory seems to be a necessary one: every element of the physical reality must have a counterpart in the physical theory." They later suggested the following as a plausible, sufficient but not necessary definition of reality: "If, without in any way disturbing a system, we can predict with certainty (i.e., with probability equal to unity) the value of a physical quantity, then there exists an element of physical reality corresponding to this physical quantity (SIC)."

Nowadays quantum mechanics is considered to be complete mathematically (or epistemologically), because no addition to quantum mechanics results in a probabilistically better theory [7]. Whether or not it is complete ontologically, thus providing a complete description of physical reality is no longer a criterion [8].

The EPR argument was not about locality per se but against contextuality, the inability to perform simultaneous measurements of incompatible (non-commuting) observables. Locality was inferred from the requirement that the systems should not interact with one another in any manner.

Subsequently Bohm [9], Bohm and Aharonov [10], Bell [11], and Clauser, Horne, Shimony, Holt [12] introduced refinements to the EPR argument which brought the scenario (or a version thereof) closer to experimental scrutiny and explicitly addressed the issues of locality and hidden variables. The idea of hidden variables refers to the existence of some unknown parameter space $\Lambda$, such that all measurements $A$ and probabilities $p$ associated with an experiment are functions of values, that is, $A(x, \lambda), p(x, \lambda)$, where $x$ refers to all of the overt variables associated with the experiment. Early papers focused on deterministic hidden variables, but as Bell pointed out [11], the question of deterministic, non-deterministic, or stochastic is irrelevant; the real question is whether or not the assumption of hidden variables can explain the observations. The Bell scenario involves two quantum systems, I, II, which interact to form an entangled state, ensuring that the states of the two systems are correlated. The systems then propagate to spacelike separated locations, $X, Y$, which, if special relativity (and therefore locality) holds, should ensure that they are unable to interact with one another in any manner. Next two independent observers, conventionally Alice and Bob, are allowed to carry out measurements, Alice of system I, Bob of system II, of (usually non-commuting) observables $A, B$, respectively, each parameterized by $a, b$, respectively. After collecting their data, Alice and Bob then determine various correlations among their measurements and then test these results against a specific inequality, namely $-2 \leq E\left(a^{\prime}, b^{\prime}\right)+E\left(a^{\prime}, b^{\prime \prime}\right)+E\left(a^{\prime \prime}, b^{\prime}\right)-$ $E\left(a^{\prime \prime}, b^{\prime \prime}\right) \leq 2$ where $E(x, y)$ is the expectation value of the product of the outcomes of measurements of the two systems when Alice's observable setting is $x$ and Bob's observable setting is $y$ ([13], chapter 8).

Bell [11], Jarrett [14], and Shimony [13] emphasized that one of the key components of the argument leading to the inequality is that the probability distribution given by the hidden variables must satisfy a factorizability condition. Following Shimony [13] and Jarrett [14], let $p^{i}(x / k, a, b)$ denote the probability of observer $i$ measuring outcome $x$ given complete state $k$, Alice's setting $a$ and Bob's setting $b . p(m, n / k, a, b)$ is the joint probability when Alice obtains measurement $m$ and Bob obtains measurement $n . p^{i}(x / k, a, b, y)$ is the conditional probability when the second observer obtains measurement $y$. Jarrett defined two independence conditions:

Parameter Independence

$$
\begin{array}{r}
p^{i}(m / k, a, b)=p^{1}(m / k, a) \\
p^{2}(n / k, a, b)=p^{2}(n / k, b)
\end{array}
$$

Outcome Independence

$$
\begin{aligned}
& p^{1}(m / k, a, b, n)=p^{1}(m / k, a, b) \\
& p^{2}(n / k, a, b, m)=p^{2}(n / k, a, b)
\end{aligned}
$$

Jarrett showed that the assumption of both independence conditions leads to the factorizability condition: $p(m, n / k, a, b)=$ $p^{1}(m / k, a) p^{2}(n / k, b)$. This condition is an essential component of most hidden variable arguments [10,11, 15-18]. When true, the hidden variables are non-contextual with a Kolmogorov probability structure, unlike quantum mechanics which has a non-Kolmogorov probability structure by virtue of the Born rule. All hidden variable arguments assume factorizability. Assuming factorizability, Fine [19, 20] showed that the presence of deterministic LNHV implies the existence of a joint probability distribution for even non-commuting observables, violating the predictions of quantum mechanics.

Kolmogorov [21] himself emphasized that probability theory was fundamentally a contextual theory. Probability distributions were context dependent. Fine [20] developed some criteria for when a joint probability distribution exists but years earlier Vorob'ev [22] had presented a complete set of criteria for the existence of joint distributions in the general case and several examples where his criteria failed to be satisfied. This clearly showed that the factorizability condition is an assumption, not a necessity. The fundamental question is whether hidden variables are non-contextual (factorizable) or contextual. 
Another approach to the EPR scenario has focused upon contextuality directly. EPR [1] and others [23] define realism to mean that every element of reality possesses, a priori, a definite value for every possible observable. Contextuality asserts that not all observables can have pre-existing values. Realism is then equated with non-contextuality. A succession of ever more powerful results (von Neumann [24], Gleason [25], Mackey [26], Kochen-Specker [27], Mermin [28]) have shown quite conclusively that quantum mechanics, in its Hilbert space formulation, is a contextual theory. Dispersion-free measures do not exist, so that it is impossible through acts of measurement to assign definite values to all possible observables to single physical entities. If realism is equivalent to non-contextuality, then quantum mechanics shows that realism does not exist. One is left with Wheeler's famous dictum that "no phenomenon is a phenomenon until it is an observed phenomenon" [29], so that there is no external reality; the actions of an observer cause reality to manifest. The realist perspective attempts to avoid falling down this particular philosophical rabbit hole.

It took nearly 50 years to develop the technology to allow testing of these theories. Most experiments have involved entangled photons although a few have used entangled electrons [30-38]. Since the first experiments of Aspect and Grangier [30] showed that the Bell inequality was indeed violated, a number of possible "loopholes" have been proposed related to experimental factors such as detector accuracy, propagation losses, detector distance, superdeterminism, all of which have been eliminated by subsequent experiments [31-36]. Even free will have been challenged [39]. Bell's inequality has been violated in all of these experiments to a statistical level of at least 11 standard deviations, and any presumed non-local influence must propagate with a speed of at least 50,000c. Bell type experiments have now become school demonstrations [37]. There is now an experiment which visualizes correlations referred to in Bell's theorem [38].

For those who accept Bell's argument and its variations, the issue would appear to be put to rest. LNHV do not exist and reality, if it even exists, is non-local.

\section{THE ARGUMENTS AGAINST THE ARGUMENTS AGAINST LOCAL HIDDEN VARIABLES}

Or is it? As in every walk of life, things are not as simple as they first appear.

Khrennikov [40], drawing on work of Hertz and Boltzmann, divides theories into two general kinds: descriptive, and observational. Descriptive (ontological) theories attempt to describe the entities (causes) that give rise to observed phenomena. Observational (epistemological) theories attempt to merely provide a predictive framework for these same phenomena. Quantum mechanics is, mostly, an observational theory. The arguments against local hidden variable described above are framed within an observational framework.

The Bell scenario involves three distinct stages: interaction, propagation, measurement. The literature has focused primarily upon the measurement stage, simply assuming the first two stages as given. Nevertheless, the derivation of the inequalities depends upon assumptions made regarding these initial stages. Quantum mechanics is not involved in this derivation. Experiments have been performed which show that the inequalities are violated. Logically then, there must be errors in the assumptions leading to the inequalities. This is not necessarily a vindication of quantum mechanics.

From an observational/epistemological perspective the key problem is the assumption of factorizability of the probability associated with whatever variables are assumed to be present in the description of the measurement situation. A factorizable probability leads inevitably to a joint distribution for the measurements, regardless of whether they are compatible or complementary. Is the assumption of factorizability necessary for any model of local realism?

Khrennikov [40], following earlier work of Landau [41], constructed a quantum mechanical analog of the $\mathrm{CHSH}$ inequality. Given 4 observables, $A_{1}, A_{2}$ for system 1 and $B_{1}, B_{2}$ for system 2 , he considered:

$<\mathcal{B}>=\frac{1}{2}\left(<A_{1} B_{1}>+<A_{1} B_{2}>+<A_{2} B_{1}>-<A_{2} B_{2}>\right)$. After some algebra he obtained the Landau identity $\widehat{\mathcal{B}}^{2}=1-$ $\frac{1}{4}\left[\hat{A}_{1}, \hat{A}_{2}\right]\left[\hat{B}_{1}, \hat{B}_{2}\right]$. If either the A or B operators are compatible, then $|<\mathcal{B}>| \leq 1$. He then showed that there exists a quantum state such that $\|\widehat{\mathcal{B}}\|^{2} \geq(1+\mu)>1$ so that quantum mechanics violates the above inequality. Khrennikov argued that the quantum analog of the $\mathrm{CHSH}$ inequality measures the degree of incompatibility among the observables being measured on each system and is not a reflection of nonlocality. Similarly, Cabello [42] has demonstrated formally that the generalized Bell inequality and Kochen-Specker contextuality are equivalent in quantum mechanics. Nieuwenhuizen [43] also examined the $\mathrm{CHSH}$ inequality and showed that the probability measures required to calculate the various correlation functions were subject to contextuality effects, so that no joint probability distribution, required to make meaningful the resulting inequality, exists. He referred to this as the contextuality loophole, and also argued for its universality. Kupczynski [44] advocates for an purely epistemological, ensemble interpretation of quantum mechanics, and also argues that the Bell argument is invalid because it fails to take into account the contextual nature of the probability distributions associated with these ensembles.

Khrennikov has developed an extension of Kolmogorov probability theory called contextual probability theory [45]. Contextual probability theory does for probability theory what non-Euclidean geometry did for geometry. The point of departure from Kolmogorov probability is the sum rule, which takes the form $p_{C}^{b}(\beta)=\sum_{\alpha} p_{C}^{a}(\alpha) p_{\beta \mid \alpha}+$ $2 \lambda(\beta \mid \alpha, C) \sqrt{\prod_{\alpha} p_{C}^{a}(\alpha) p_{\beta \mid \alpha}}$ where

$\lambda$ is the probabilistic measure of interference and the $p$ terms are various conditional probabilities over contexts (C) and observables $a, b$. In Kolmogorov probability, $\lambda=0$, otherwise $\lambda$ can be a trigonometric function or a hyperbolic function. Contextual probability has been applied to a number of classical level phenomena in biology, psychology, and economics [45-51] and to the Bell situation [52, 53]. The appearance of 
non-Kolmogorov probability at a classical level demonstrates empirically that the assumption that any probability associated with local hidden variables must be Kolmogorov is prima facie false. Hidden variables may be non-contextual or contextual at both classical and quantum levels. This does not necessitate non-locality or a failure of realism.

Dzhafarov et al. [54], Dzhafarov and Kon [55], and Dzhafarov and Kujala [56] has presented an alternative approach to that of Khrennikov termed Contextuality by Default. Following the notation in [57], each random variable is associated with the quantity $q$ being measured and the context $a$ within which the measurement is made, and denoted, $R_{q}^{a}$. Consider two measurements, $q, q$ ' and two contexts $a, b$. For a fixed context $a$, the pair $R_{q}^{a}, R_{q^{\prime}}^{a}$ is termed bunch, representing the collection of measurements associated to a specific context. It is reasonable to believe that such a pair is jointly distributed. For a fixed measurement $q$, the pair $R_{q}^{a}, R_{q}^{b}$ is termed a connection for $q$.

The most basic form of contextuality occurs when no joint distribution can be found for a connection. In such a case they are said to be inconsistently connected. This is the situation of contextuality by default. Dzhafarov considers this to be the most trivial form of contextuality since it is so ubiquitous. Dzhafarov has developed a more restricted notion of contextuality in line with contextuality in physics. He considers couplings between bunches. For example, given two bunches $R_{q}^{a}, R_{q^{\prime}}^{a}$ and $R_{q}^{b}, R_{q^{\prime}}^{b}$, a coupling is a set of jointly distributed random variables $(A, B, X, Y)$, subject to certain constraints, such that $(A, B)$ is distributed as $R_{q}^{a}, R_{q^{\prime}}^{a}$ and $(X, Y)$ is distributed as $R_{q}^{b}, R_{q^{\prime}}^{b}$. The constraints involve $A, X$ and $B, Y$ which correspond to measurements of $q$ and $q$, respectively. A measurement $q$ is considered to be context independent if among all couplings $(A, B, X, Y)$, we have $\operatorname{Pr}(A \neq X)=0$. It can be shown that such a coupling may not exist even if the system is consistently connected.

Now consider all couplings $(A, X)$ for just the connection $R_{q}^{a}, R_{q^{\prime}}^{b}$ and find the minimal value $m^{\prime}$ for $\operatorname{Pr}(A \neq X)$. Then consider the global coupling $(A, B, X, Y)$ and again find the minimal value $m$ for $\operatorname{Pr}(A \neq X)$. If $\mathrm{m}=\mathrm{m}$ ' the system is noncontextual and if $m>m$ ' then the system is contextual. This form of contextuality is analogous to that found in physics and gives rise to similar types of inequalities.

Contextuality by default has been observed experimentally [57]. Moreover, two recent studies [58, 59] have demonstrated the strong form of contextuality in a social psychological setting [58] and in individuals [59]. Contextuality in the form observed in quantum mechanical settings is thus not unique to the quantum domain but can occur in classical settings as well. Dzhafarov and Kon [55] have analyzed the Bell scenario within the contextuality-by-default model, and showed that it can be understood using wholly classical (albeit contextual) probability theory.

Dzharfarov and Kujala [60] applied Contextuality by Default analysis to the double slit experiment. They pointed out that "Contextuality or non-contextuality is a property of a system of random variables representing an empirical situation rather than of the empirical situation itself." They presented a very general model of the two slit situation, using Kolmogorov probability together with the addition of a context parameter, much as Kolmogorov originally argued [21], and obtained the usual statistics. Dzhafarov argued that the Bell argument is not about the nature of physical reality but rather about the failure to take context effects into account when creating a Kolmogorov type probability model of a situation.

The role of context in probability theory has not always been ignored. Kolmogorov [21] and von Mises [61] understood that probability theory was contextual. Pitowski $[62,63]$ analyzed the Bell situation and presented a model based on a form of contextual probability. In his model "The relative frequencies violate Bell's inequality the way they do because the locality principle is true" (SIC) [63]. Later, Pitowski [64] developed a deterministic model of spin statistics using the concept of nonmeasureable sets. He argued [63] that quantum mechanics is essentially a probability theory, which in Khrennikov's language would be viewed as a trigonometric contextual probability theory. Gudder [65] applied a generalized probability theory similar to that of Pitowski to the problem of spin statistics and showed that such a model was compatible with local hidden variables. Gudder [66] had already shown that a hidden variable model of the Bell scenario was possible so long as contextual hidden variables were used, a line of thought supported a few years later by Ballentine [67]. Local contextual hidden variable models have also been developed by Durdevic $[68,69]$. Recently Griffiths [70], using a coherent histories approach, has reaffirmed that quantum mechanics is a local theory, the inequalities are a consequence of the contextuality of quantum mechanics, and the correlations that are detected in a typical Bell experiment arise due to a common quantum cause [71]. These models seem to have been ignored in the mainstream literature. The belief that the probability theory of the classical world is necessarily Kolmogorov and non-contextual has achieved the status of dogma, and it has proved extremely difficult to disabuse people of this.

A different approach was proposed by Palmer [72] who developed a deterministic model of the spin scenario but where, crucially, there was a non-linear dynamics in place. The consequence of this was the impossibility of forming the correlation functions required for the Bell inequality. Experimentally, correlations can always be calculated but they need not be meaningful [73]. He suggested that the inequalities said nothing about the nature of reality, since the correlation functions involved did not exist.

Remarkably, contextual hidden variable theories have not gained much traction within the foundations community. In spite of their ability to reproduce the quantum mechanical results while preserving locality, they have, for the most part, been ignored in favor of the quasi-mystical notion of non-locality. Shimony ([13], chapter 10) defined two types of contextual hidden variables: environmental, which include experimental conditions, and algebraic, referring to models on quantum logics or lattices. He rejected both types of contextual hidden variables of the environmental type ([14], chapter 10), arguing that they would still satisfy a factorizability condition, but without proof. Shimony appeared to reject locality, evading special relativity by 
his "passion at a distance." He argued that a breach of outcome independence did not imply superluminal signaling, but outcome dependence reflects contextuality, not necessarily non-locality.

\section{LOCAL REALISM NEED NOT IMPLY NON-CONTEXTUAL LOCAL HIDDEN VARIABLES}

If the observational approach to theory suffices, then it appears clear that measurement is a contextual act. Non-disturbing, noncontextual, "objective" measurements do not exist universally. The classical notion of objectivity does not hold true. This does not, however, imply that an act of measurement creates reality, merely that reality may be altered by such an act. Reality appears to be interactive, and thus characterized by a weak form of subjectivity. This appears easier to accept than non-locality, since evidence for contextuality is all around us, while evidence for non-locality is profoundly lacking. Griffiths writes [70] "To be sure, those who claim that instantaneous non-local influences are present in the quantum world will generally admit that they cannot be used to transmit information; this is known as the 'no-signaling' principle, widely assumed in quantum information theory. This means that such influences (including wave function collapse) cannot be directly detected in any experiment. The simplest explanation for their lack of influence is that such influences do not exist."

Pusey, Barrett and Rudolph [74] have argued that the wave function is ontological, and experiments visualizing Belltype non-local behavior [38], quantum jumps [75], quantum measurement processes [76], quantum trajectories [77], quantum wave functions [78], and single photons [79] would seem to support this. Evidence that quantum jumps [75] and quantum measurements [76] evolve over a period of time suggests that there is an actual "something" out there corresponding to such behavior. This suggests that a purely observational theory is inadequate and a descriptive theory is also needed to explain contextuality and the presence of long range correlations.

The dominant viewpoint, however, is that the wave function is merely epistemological, and that quantum mechanics deals only with the statistics and behaviors of ensembles. The ability to carry out single photon and single particle experiments demonstrates that this is not a necessity $[80,81]$. The ensemble approach focuses on the density matrix $\rho$, defined as $\rho=\sum_{i} p_{i}\left|\phi_{i}><\phi_{i}\right|$ where the $\mid \phi_{i}>$ are pure states [82]. Moreover, the density matrix involves two different kinds of probability: an explicit classical probability in the form of the real valued $p_{i}$ and an implicit non-Kolmogorov probability in the form of the squared modulus of the complex valued amplitudes of the pure states. These two considerations suggest that ensembles should be treated ontologically as supervening on pure states. Thus, questions of ontology should reference pure states.

The challenge in finding an ontological model of pure states in quantum mechanics lies not with the measurement problem (for which a detailed model within the usual quantum mechanical framework has been proposed $[83,84]$ ) but rather in providing an ontological understanding of superposition. A naïve attribution of measurements to a single particle in a superposition state leads to confusion and paradox, causing many to abandon an ontological interpretation of the wave function and sometimes reality itself.

Norsen [85] has pointed out that dispensing with the idea of realism, broadly considered, results in the end of scientific inquiry, because without some notion of a "reality," what is it that scientists have to talk about? But must realism be identified with classical objectivity? Zeilinger et al. write "objects have physical properties independent of measurement (the assumption of realism)"([34], p. 250401-1). But this is just the definition of non-contextuality. This definition of realism seems to beg the question.

Rosen [86] suggested that physics' focus on inanimate matter has resulted in an unnecessarily limited world view. Experience with emergent systems in biology, psychology, and economics $[87,88]$ has demonstrated that many naturally occurring systems and phenomena are transient, open, multiscale, emergent, generated and generative, contextual, and subjective. It is doubtful that anyone seriously considers these systems to be "real" only as a result of "observation." It is not realism that needs to be abandoned-rather concepts such as ideal object, ideal non-disturbing measurement, and non-contextuality must go.

A metaphysics with subjective elements was proposed by Whitehead [89] nearly a century ago. It is a process model of reality which emphasizes its transient, generated, generative, emergent, and contextual features. Several authors have proposed process models of physics [2-6, 90-99] and even Shimony [13] wrote about Whitehead's idea of process.

Consider an alternative definition of realism. To begin, why is it necessary that an element of reality possess a priori all of the properties that can be measured on it? Measurement is an act, and always involves an interaction between a system and a measuring apparatus. It is generated in the moment as the interaction takes place. There is no need to assume that something corresponding to this measurement exists in the system prior to its interaction with the measurement apparatus. It is only necessary that the system possesses the potential to determine such a measurement when it interacts with a measurement apparatus. It is equally unreasonable to assert that nothing exists prior to the interaction with the measurement apparatus. The system must exist or what exactly does the measurement apparatus interact with? Moreover, the interaction with the system results in a systematic difference which ensures that only particular measured values are returned with particular frequencies. If the measurement apparatus creates the measurement, then why just these values and no others? The system must possess a potentiality which becomes realized in any interaction with a measurement apparatus.

Why too is it necessary that for something to be real it must be knowable to a human observer. Quantum mechanics appears obsessed with the idea of measurement, yet events occur in nature without any obvious "measurement" taking place and without any "observer." A theory of natural processes should, reasonably, describe the evolution of such processes as they occur "in vivo," and not merely "in vitro," in a laboratory. Moreover, 
such a theory should be able to deal with single entities, not merely ensembles.

At a bare minimum, any entity must possess some propensity to determine a difference in the state or future history of at least one of the entities that it interacts with. For, if something is thought to exist, but in any interaction with anything whatsoever, in any manner and for all time, it never determines any difference whatsoever, then it might just as well not exist, since its existence will never be noted. Of course an entity may never have an opportunity to interact with another entity, so it is not essential that a difference be realized, only that the entity possesses a propensity to determine a difference, should a suitable interaction occur. These two considerations suggest the following minimalist definition of realism:

An entity is real if it exhibits a propensity to determine a systematic difference. An entity or phenomenon can determine a difference in only a single interaction for a single time, or across many interactions over multiple times. It may determine the same difference at multiple times, or it may determine different differences on different occasions, but these should be systematic in some manner. With respect to the same initial conditions, the difference may be deterministic (fixed single value), non-deterministic (fixed set of values), or stochastic (fixed probability distribution). There is no need for an observer, particularly a human observer. Previous comments aside, if this potentiality is never realized then its reality is rather moot. Thus, there is a need for an entity with which it can interact so as to realize this potentiality and thus register its reality. It may be that this additional entity is itself, and that selfinteraction might provide the most basic interaction realizing a potentiality, perhaps that of bare existence. The realization of other potentialities requires interactions with wholly separate entities. The minimum requirement for such entities is that they can register a difference in either state or history, a concept referred to as salience [100]. There is no need for consciousness or agency.

The determination of a difference requires interaction; it is relational. Some differences may be private, specific to an individual entity (for example quarks and gluons) or public, accessible to many entities (for example photons). The idea of reality being a propensity to determine a difference has much in common with the pragmatism of John Dewey [101] and the process view of Whitehead [89]. Propensity drives home the point that it is the capacity to determine a difference which matters, not which difference it is which is determined.

A quantum system determines a potentiality to obtain certain measured values through an act of measurement. These values are only realized through an interaction with a measurement apparatus. It is not necessary that the quantum system possess these measured values, merely that it possess the propensity to determine them, if only in a statistical manner. It is a set of dispositions. Since only select measurements are made possible, it makes a difference, and by the definition proposed here, it is real. It might be associated with a particular measurement on one occasion, but this need not be the same on a subsequent occasion. That will depend upon the intervening interaction history. Properties can be real, they can be contextual, they simply need not be eternal.

Measurements are not specific properties of the system but propensities, one of which may be realized following a measurement act. The system does not possess these properties but rather, together with a suitable measurement apparatus, acts as a generator of properties. Thus, quantum systems should not be thought of as "objects" but rather as "processes." Since processes make a difference, somewhat, somewhere, sometime, processes are held to be elements of reality. They are ontological entities but with characteristics such as transience, emergence, generativity, agency, contextuality, and locality.

Some quantum properties have a universal character, such as whether their wave function is scalar, spinor, vector, tensor, or their charge, rest mass, and so on. These can be attributed to the system itself. Many other properties, however, are contextual in character and thus should be treated as generative propensities. For example, whether a quantum system is to be considered wave-like or particle-like is contextual. Indeed, Ionicioiu et al. [18] showed that the wave-particle distinction is not compatible with a non-contextual hidden variable representation. The tracks of fundamental particles are also contextual in that they do not occur in the absence of a detector. Mott [102] showed that the formation of particle tracks in a bubble chamber was an emergent feature of the interaction between the particle and the atoms in the bubble chamber. There are no tracks without the bubble chamber. The formation of tracks is a propensity of the particle, not a property of the particle.

Consider again the Bell correlations. Reichenbach [103] formulated the principle of the common cause, which states that if an improbable coincidence has occurred, there must exist a common cause. In the Bell scenario, the common cause is presumed to occur at the time at which measurements on the two systems take place. However, consider the following Gedanken experiment. Consider a source which produces a pair of spinentangled particles I, II, with opposite momenta $\boldsymbol{p}$, $\boldsymbol{p}$, which are allowed to move to locations a distance $d$ apart, where there are placed detectors for Alice and Bob. The detectors of Alice and Bob are space-like separated. The outcome of each measurement is sent to a common location at a distance $h$ from each detector where there is a recording device which measures the outcome from each detector simultaneously. A common trigger is established at a distance $r$ from each detector. When a signal from the trigger is sent, each detector makes a measurement, thus ensuring simultaneity. In the interval between trigger signals, Alice and Bob are free to alter the specific measurement being made by their detector.

The output from the source has a wave function of the form

$$
\mid \Psi\left(\mathbf{r}_{1}, \mathbf{r}_{2}\right)>=\frac{1}{\sqrt{2}}\left[\left|\phi_{1}: \rho_{1}\left(\mathbf{r}_{1}, \mathbf{r}_{2}\right)>+\right| \phi_{2}: \rho_{2}\left(\mathbf{r}_{1}, \mathbf{r}_{2}\right)>\right] .
$$


The question is: at what point in this situation is the supposed passion at distance to play out?

The unusual notation for the wave function is intentional. In part it follows Mott's [102] argument in which he emphasized that the wave function for the interaction between particle and bubble chamber fluid possessed a complex, non-separable dependence on both the particle and the atom with which it is interacting. It is also meant to emphasis the algebraic aspect of Hilbert space expressed in the bra-ket notation and the representation of this algebra by means of the wave function. This will be important later in the discussion of the Process Algebra. Note that knowledge of this wave function comes from outside Alice and Bob. From Alice's point of view, there is a single particle whose wave function is $\left|\Psi_{1}\left(\mathbf{r}_{1}\right)\right\rangle=\frac{1}{\sqrt{2}}\left(\left|\phi_{1}\left(\mathbf{r}_{1}\right)>+\right| \phi_{2}\left(\mathbf{r}_{1}\right)>\right)$, while Bob sees a single particle with wave function $\left|\Psi_{2}\left(\mathbf{r}_{2}\right)\right\rangle=$ $\frac{1}{\sqrt{2}}\left(\left|\rho_{1}\left(\mathbf{r}_{2}\right)>+\right| \rho_{2}\left(\mathbf{r}_{2}\right)>\right)$.

If they were to pool their descriptions they would assume that the combined wave function is $\left|\Psi\left(\mathbf{r}_{1}, \mathbf{r}_{2}\right)\right\rangle=\left|\Psi_{1}\left(\mathbf{r}_{1}\right)\right\rangle$ $\left|\Psi_{2}\left(\mathbf{r}_{2}\right)\right\rangle$, which is clearly inconsistent with the actual case and also with what is observed by the recorder. Why then are we so quick to assume that the entangled wave function

$$
\begin{array}{r}
\mid \Psi\left(\mathbf{r}_{1}, \mathbf{r}_{2}\right)>=\frac{1}{\sqrt{2}}\left[\left|\phi_{1}: \rho_{1}\left(\mathbf{r}_{1}, \mathbf{r}_{2}\right)>+\right| \phi_{2}: \rho_{2}\left(\mathbf{r}_{1}, \mathbf{r}_{2}\right)>\right] \\
=\frac{1}{\sqrt{2}}\left[\left|\phi_{1}\left(\mathbf{r}_{1}\right)>\right| \rho_{1}\left(\mathbf{r}_{2}\right)>+\left|\phi_{2}\left(\mathbf{r}_{1}\right)>\right| \rho_{2}\left(\mathbf{r}_{2}\right)\right]>?
\end{array}
$$

Alice and Bob will make their measurements simultaneously, at exactly the same proper times for each particle. If Alice were to carry out her measurement slightly earlier than Bob, Alice would presumably collapse the wave function of particle I and cause the wave function of particle II to collapse to the corresponding entangled state, and conversely if Bob measures first. Here, however, the measurements are carried out simultaneously. So what will happen? If nothing exists prior to the simultaneous measurements of Alice and Bob, then it is not clear at all how this is to be resolved. Alice and Bob believe that they are working with free particles yet the recorder will obtain correlated measurements. A definite difference will be observed. Thus, according to the definition of realism being considered here, a definite something must exist prior to the measurements taking place.

The entangled system exhibits a propensity to determine two simultaneous correlated measurements which make a difference, and thus it is reasonable to consider that the entangled system represents a single element of reality. The error lies in assuming that one has two quantum systems which are somehow correlated. That seems possible only through the passage of some signal between them, but in the case of simultaneous measurements, what could such a signal convey? Such a signal not only must be instantaneous, but it must also effect a choice. This seems implausible.

A simpler explanation is that there is only one system, but it produces two measurements. This could occur in one of two ways. First of all, when the entangled state is created, entangled particles are emitted in either of the two entangled states, $\mid \phi_{1}: \rho_{1}\left(\mathbf{r}_{1}, \mathbf{r}_{2}\right)>$ or $\mid \phi_{2}: \rho_{2}\left(\mathbf{r}_{1}, \mathbf{r}_{2}\right)>$, and then propagated. In that case the wave function is merely epistemological, describing a statistical ensemble of entangled particles. The second possibility is more interesting. At the moment of measurement the entangled system has the propensity to manifest either state $\mid \phi_{1}: \rho_{1}\left(\mathbf{r}_{1}, \mathbf{r}_{2}\right)>$ or $\mid \phi_{2}: \rho_{2}\left(\mathbf{r}_{1}, \mathbf{r}_{2}\right)>$, never both, with $50-50$ frequency. In either of these cases there is no need for quasimystical instantaneous signals to be passing back and forth between measurement devices.

Now suppose that the experimental situation is rescaled, so that all distances diminish by a proportion $p$. The above argument can be repeated and again, the entangled system exhibits the propensity to manifest either state $\mid \phi_{1}: \rho_{1}\left(\mathbf{r}_{1}, \mathbf{r}_{2}\right)>$ or $\mid \phi_{2}: \rho_{2}\left(\mathbf{r}_{1}, \mathbf{r}_{2}\right)>$, never both. It follows that this must hold true for every moment of time. Since, at every such moment of time, the entangled system has the propensity to determine a difference, there must be an element of reality present at each moment of time. The entangled system is thus the generators of these momentary propensities.

Since the wave function appears to be at least partially ontological, the simplest explanation for this propensity is that at each moment of time, the entangled particle system actually manifests either state $\mid \phi_{1}: \rho_{1}\left(\mathbf{r}_{1}, \mathbf{r}_{2}\right)>$ or $\mid \phi_{2}: \rho_{2}\left(\mathbf{r}_{1}, \mathbf{r}_{2}\right)>$, never both, and that the state may change from moment to moment.

If one believes in the principle of continuity then this is quite problematic and perplexing. If time is continuous then the evolution of the entangled system must be neither smooth, nor continuous. For if $\mathrm{H}$ is the Hamiltonian and time evolution is given by the usual operator, $U(t)=\exp [-i t H / \hbar]$, then assume that there is some time interval $\left[t_{1}, t_{2}\right]$ on which the entangled state is constant. Then $U(t)=I$ for every $t \leq t_{2}-t_{1}$, and hence $U(t)=I$ for all $\mathrm{t}$. It appears that one must abandon the principle of continuity. Bancal et al. [17] and Gisin [104] and colleagues addressed this problem in the context of a Bell scenario. They studied the case of 4 quantum observers and, by assuming the principle of continuity and a constant, finite but unspecified superluminal speed $v(c<v<\infty)$ of propagation of any hidden signals, were able to find an inequality involving various correlated measurements, as well as a quantum state which violated the inequality. They concluded that either the principle of continuity must be violated, or superluminal signaling must be possible. Gisin wrote "Note that the finding of such a speed would falsify both quantum theory and relativity, a result not many physicists are willing to envisage" [104, pg 10] thus favoring abandoning the principle of continuity. Bancal et al. wrote "This gives further weight to the idea that quantum correlations somehow arise from outside spacetime, in the sense that no story in space and time can describe how they occur" ([17], pg. 4).

The theory of special relativity has survived multiple experimental tests and has yet to be violated. The principle of continuity, however, is frequently violated at smaller scales, so why should it not be violated at the smallest scale? This would appear to provide far less of a shock to our conceptual system than the assumption of instantaneous transmission of undetectable signals. Indeed a recent paper argues that there is an upper limit to the frequency of any physical process, including 
any "clock," with the shortest temporal interval on the order of $10^{-33} \mathrm{~s}$ [105].

Local realism, according to the new definition, appears to be perfectly tenable provided one accepts contextuality and abandons the principle of continuity. The remainder of this paper is devoted to describing just such a locally realist model of non-relativistic quantum mechanics (NRQM) without hidden variables.

\section{PROCESS AND THE PROCESS ALGEBRA MODEL}

One way to implement a descriptive theory together with the new definition of realism is through the concept of process. This has its origins in the writings of Heraclitus and Siddhartha Gautama and its modern version in Whitehead's process theory. A propensity to determine differences can be accommodated if those differences are generated. A process is viewed as a generator of primitive events called actual occasions, the base elements of reality. By the new definition of realism, if a process is responsible for determining differences, manifesting as actual occasions, then a process must be accorded the same ontological status, that is, the same degree of reality, as those occasions.

Whitehead considered a process to be a sequence of events having a coherent temporal structure in which relations between the events are more fundamental than the events themselves. Whitehead viewed process as being ontologically prior to substance and becoming to be a fundamental aspect of being. Becoming is fundamental to process, and fundamental to becoming is transience. In process theory events have a transient existence, coming into being, manifesting briefly, then fading away. Each actual occasion exists only long enough to prehend the realities of the previous events and to form a response to them, thereby immediately passing out of existence and becoming data for subsequent events. Actual occasions, the basic elements of reality, are held to be inseparable occasions-in-connection, giving reality a holistic aspect. The act of prehension underscores that information plays a fundamental role in the unfolding of reality, and that in particular, it is meaning that is necessary to give rise to coherence among events [90]. Reality is emergent, arising out of a lower level of actual occasions as are the fundamental physical entities, which are viewed as emergent configurations of actual occasions $[13,89]$.

Actual occasions do not occur in space-time, nor do they move in space-time. Instead, actual occasions form space-time. They are the primitive "events" upon which natural entities supervene and from which they emerge. Processes, being generators of actual occasions, are thus generators of space-time itself. Logically, processes do not exist within space-time, they stand outside of it, thus fulfilling the suggestion of Bancal et al. [17].

In keeping with the descriptive perspective, actual occasions constitute the signs by means of which processes implement their propensity to determine differences. An actual occasion marks a specific expression of this propensity, determining one of whatever many differences the process may determine. Actual occasions thus mark whichever difference is being determined, in the moment, and this difference may vary from moment to moment.

The idea of process depends crucially upon the idea of becoming, and that in turn requires a transient now. Such a concept is thought to be incompatible with special relativity, but this is a misunderstanding of what special relativity implies. As Wigner pointed out [106], what special relativity demonstrated is the non-existence of global frames of reference. All global frames of reference are mathematical fictions. Simultaneity, and thus a transient now, can exist, but co-moving observers within the universe will not agree about this. Denying simultaneity is another example of misplaced omniscience. Reality may unfold according to a transient now even if human observers cannot detect it. Several authors have argued that it is not the block universe which is a necessity but rather some form of presentism [107-110].

Similar to the actual occasions that they generate, processes shift between periods of activity and inactivity. While active, they express a propensity to determine differences, manifesting in distinct attributes and functionality. Processes interact with one another according to their attributes and functionalities and the actual occasions that they manifest, and these interactions are triggered by the manifesting of particular actual occasions.

Process ideas can be seen in Finkelstein's quantum relativity [92, 95], Noyes's bit-string physics [94], Bastin and Kilmister's combinatorial physics [96], Hiley's process physics [93], Cahill's process physics [97]. Emergent models of physics include Nelson [111], Adler [112], Levin and Wen [113], and two time models such as stochastic quantization [114] and Bars' two time physics [115].The process algebra model has many roots: Sorkin's causal sets [116] (whose basic elements could be reinterpreted as actual occasions), Lee's discrete time dynamics [117], Kempf's interpolation model of QFT [118] (which suggested that NRQM could be emergent from a discrete space). Related models include the cellular automata models of ' $t$ Hooft [119] and Elze [120], which appear to be special cases of process algebra models. Trofimova $[98,99]$ has proposed several process algebra based formalisms for describing the principles of transience which govern processes in functional constructivism. Her approach to process algebra uses several functional differentiation classes, a concept of "performance" and several universal process-trends. It applies particularly to complex, adaptive, multiscale systems. Her work has provided much inspiration for the author.

The next few pages describe the process algebra model in terse detail. A more leisurely discussion can be found in $[2,4]$. The process algebra model considered here views quantum mechanics as an (incomplete) effective theory, being the asymptotic limit as spatiotemporal scales become infinitesimal. The Hilbert space formalism is considered to be mathematically coarse, blurring the distinction between ontological and epistemological, and leading to a great deal of unnecessary confusion.

Since von Neumann, the use of the language of Hilbert spaces for formulating NRQM has become dogma [24]. The Process Algebra model starts from the realization that the Hilbert space of NRQM is a reproducing kernel Hilbert space [121]. Given a reproducing kernel Hilbert space $H(X)$ with base space $X$, one can find a discrete subspace $Y$ of $X$ (sampling subspace), and a 
Hilbert space $H(Y)$ on $Y$, such that each function in $H(Y)$ can be lifted to a function in $H(X)$ via interpolation. Interpolation means that if $\Psi(z)$ is a function in $H(X)$, then for each $y \in$ $Y$ there exists an interpolation function $\Psi_{y}(z)$ on $\mathrm{H}(X)$ such that $\Psi(z)=\sum_{y \in Y} \Psi(y) \Psi_{y}(z)$. In general there are usually an infinite number of these sampling subspaces. The interpolations functions are not unique. They are usually chosen by reason of goodness of fit. In the case that the subspace $Y$ has the form of a regular lattice the interpolation functions may be taken to be sinc functions $(\sin x / x)$ [90]. If the subspace has an irregular structure with density matching the Beurling density [122], FechtingerGröchenik interpolation theory may be used instead [121, 123]. Interpolation does not reproduce all functions on $H(X)$ but rather a more limited set of band-limited functions, that is, functions whose Fourier transform is limited to a bounded set, ensuring the existence of a natural ultraviolet cutoff.

In interpolation theory, $H(X)$ is considered to be fundamental while $H(Y)$ is derived, a result of a sampling procedure. The Process Algebra model reverses this relationship. The discrete subsets $Y$ are considered to be fundamental, their elements representing the actual occasions of Whitehead's process theory. The elements of $H(Y)$ are the ontological wave functions, and the elements of $H(X)$ are derived (emergent) through an (arbitrary) interpolation procedure. The elements of $Y$ are considered to be generated by process, $\mathbf{P}$, and the value $\Psi(y)$ assigned to a point $y$ in $Y$ is also generated by $\mathrm{P}$ by causally propagating specific information from prior actual occasions to nascent actual occasions by means of a causal propagator, $\mathbf{K}$. The resulting wave function $\Psi(z)=\sum_{y \in Y} \Psi(y) \Psi_{y}(z)$ is thus emergent. The discrete subsets are called causal tapestries and their individual points are called informons. The triad of prior causal tapestry, process, nascent causal tapestry forms a compound present.

It is essential to understand that all of the physics takes place on the causal tapestry $Y$. The space $X$ is treated as emergent. Interpolation may be used to recover all of the physics on the emergent space $X$. The informons represent the fundamental elements of reality. Information among the elements is propagated only in a locally causal manner. Note that these informons do not constitute hidden variables. There is no additional parameter space associated with these informons. They constitute the fundamental elements of space-time, and their causal relationships are space-time. Moreover, they are the wave function. The wave function $\Psi(\mathbf{z})$ is not a function of these informons, it is these informons. The process model thus possesses local realism without hidden variables. This is an important distinguishing feature from other contextual hidden variable models. Moreover, the wave function in the process algebra framework is both ontological and epistemological. More about this will be discussed later. As informons are the formal representation of actual occasions, it is important to note that informons do not move in space-time. Information propagates, informons do not. They merely come into existence and then fade away. Informons may be generated in a discontinuous manner. This does not violate special relativity since no information is transferred between the informons that comprise a causal tapestry, only causally from prior to nascent causal tapestry.

A simple visual analogy might help. Think of space-time like an LED display, with each active LED element representing an actual occasion. These LED elements are lit at random, but on an ultrafast, imperceptible time scale. The resulting image represents our observable reality. Processes are represented by the signal which determines which elements are lit.

Each informon takes the form: $[n]<\mathbf{p}_{n} ; \mathbf{m}_{n}: \phi_{n}(\mathbf{z})$; $\Gamma_{n}>\left\{\mathbb{G}_{n}\right\}$ where

1) $n$ is a heuristic mathematical label,

2) $\mathbf{p}_{n}$ is a structured set of intrinsic properties,

3) $\mathrm{m}_{n}: \phi_{n}(\mathrm{z})$ is a pair of extrinsic properties,

4) $\Gamma_{n}$ is the local coupling effectiveness,

5) $\mathbb{G}_{n}$ is a causally ordered collection of informons, with causal metric $\rho$, called the content (based on an idea of Markoupoulou [124]). The union of content sets over all informons in the causal tapestry must itself form a causal set $[116,124,125]$. The causal distance is related to the depth of the causal structure, and the delay in formation flow (important in the case of non-zero rest mass).

The brackets [,],<,>,\{,\} are simply delimiters.

The local process strength at an informon $n$ is given as $\Gamma_{n}^{*} \Gamma_{n}$. The information residing in the informons of the content is utilized by the generating process to create the informon. The intrinsic properties $\mathbf{p}_{n}$ are attributed to the generating process $\mathbf{P}$ and imparted to each informon generated by $\mathbf{P}$. The extrinsic properties are unique to each informon but are frame dependent. Each informon $n$ is interpreted as a point $\mathbf{m}_{n}$ (causal manifold interpretation or embedding) in some causal manifold $\boldsymbol{M}$. Its content set $\mathbb{G}_{n}$ causally embeds into $\boldsymbol{M}$. Each causal tapestry forms a causal antichain in $\boldsymbol{M}$, and thus represents a discrete sampling of a spacelike hypersurface in $\boldsymbol{M}$. Each informon $n$ is associated with a local Hilbert space interpretation of the form $\phi_{n}(\mathbf{r})=\Gamma_{n} f_{n}\left(\mathbf{r}, \mathbf{m}_{m}\right)$, the Hilbert space $H(\boldsymbol{M})$ being that over the causal manifold $\boldsymbol{M}$. Each causal tapestry I is associated with two different maps: a tapestry realization (or allowing a slight misnomer, a tapestry "wave function") of the form $\Omega(n)=\Gamma_{n}$, and a global Hilbert space interpretation over the causal manifold of the form $\Psi(\mathbf{r})=\sum_{n \in I} \Gamma_{n} f_{n}\left(\mathbf{r}, \mathbf{m}_{n}\right)$. When the informons of a causal tapestry embed into the causal manifold as a discrete lattice, it is possible to replace each $f_{n}\left(\mathbf{r}, \mathbf{m}_{n}\right)$ by a spatial translation $\left(T_{\mathbf{m}_{n}} f(\mathbf{r})=f\left(\mathbf{r}-\mathbf{m}_{n}\right)\right)$ of a single generic sinc function $g(\sigma, \mathbf{z})=\sin \sigma \mathbf{z} / \sigma \mathbf{z}$, so that $\Psi(\mathbf{r})=\sum_{n \in I} \Gamma_{n} T_{\mathbf{m}_{n}} g(\sigma, \mathbf{r})$. The lattice spacing must be consistent with the Beurling density [122]. Maymon and Oppenheim [126] have shown that non-uniform embeddings still provide a highly accurate approximation using sinc interpolation so long as the spatiotemporal density is large enough. A more realistic model requires the use of non-uniform embeddings and more sophisticated interpolation techniques, such as FechtingerGröchenik theory [121].

A tapestry realization is analogous to a space representation of a wave function. There is a dual causal tapestry which can be 
formed using the duals of the content sets and which gives rise to an analog of the momentum representation, but this construction will not be needed here.

A process generates individual informon in a series of short rounds, collectively forming a round, in which information is propagated. A causal tapestry is generated in a series of rounds, forming a complete generation. Processes possess three additional intrinsic characteristics:

1) $r$, the number of prior informons whose information is incorporated into an informon $n$. It is also the cardinality of $\mathbb{G}_{n}$, and the number of short rounds needed to form $n$.

2) $\mathrm{N}$, the number of informons in each generation, and thus the number of rounds and the cardinality of the causal tapestry I.

3) $R$, the number of informons generated per round. A primitive process has $\mathrm{R}=1$. Otherwise the process is compound.

Process properties include invariants such as charge, rest mass, mathematical type (scalar, spinor, vector, tensor, real, complex, quaternion etc.) as well as conserved quantities such as energy, momentum, angular momentum. Conserved quantities are not considered fundamental but rather result from symmetries of the causal propagator [90,127]. A process can possess a well-defined energy or momentum but there is no dispersion free measure because the informons which are generated by the process are dispersed in space-time. The Heisenberg Uncertainty relations still hold.

The action of a process involves:

1) The assignment of a new informon label

2) The assignment of property set $\mathbf{p}_{n}$

3) The assignment of causal relations and distances to prior informons

4) The assignment of a content set $\mathbb{G}_{n}$

5) The propagation of information from prior informons.

6) Determination of local coupling effectiveness by propagating the local coupling effectiveness from each informon in $\mathbb{G}_{n}$ forward to $n$ according to the rule $\Gamma_{n}=\sum_{m \in I} K(n, m) \Gamma_{m}$ where the propagator $K$ will depend upon the causal distance $\rho(n, m)$. The propagator will be determined by particle and interacting potentials.

The dispersion of informons and subsequent causal diffusion of their information is consistent with the interpretation of the Schrödinger equation as describing a diffusion process [128]. One particular version of the process algebra model can be shown to be equivalent to Feynman path integrals $[2-4,129]$, but without the interpretation of motion along all possible paths. Moreover, it can be shown that if the propagator is relativistically invariant, then the generation of informons is also relativistically invariant [2-6].

Interactions between processes are conjectured as being triggered by the generation of informons according to the compatibility between the processes. Compatibility between interacting complex systems is an idea first proposed by Trofimova [130]. In the current context it can be thought of as a generalization of the idea of coupling factors. Compatibility $\Xi(\mathbf{P}, \mathbf{M})$ is conjectured to be a function of fixed factors such as mass, charge, coupling constants, and of the local compatibilities. The probability of an interaction taking place $\Pi(\mathbf{P}, \mathbf{M})$ is in turn a function of the compatibility, $\Pi(\mathbf{P}, \mathbf{M})=\chi(\Xi(\mathbf{P}, \mathbf{M})$. The precise form of these functions depends upon the particular case. The Born rule is expected to arise from these interactions and from the compatibility, but a precise derivation is not yet in hand. If one naively applies the Born rule, then probability will be proportional to the local process strength. If so then it will be non-Kolmogorov by virtue of the presence of interaction terms

$$
\begin{gathered}
\Gamma_{n}^{*} \Gamma_{n}=\sum_{m \in I} K(n, m)^{*} \Gamma_{m}^{*} \sum_{m^{\prime} \in I} K\left(n, m^{\prime}\right) \Gamma_{m^{\prime}} \\
=\sum_{m \in I} K(n, m)^{*} K(n, m) \Gamma_{m}^{*} \Gamma_{m}+ \\
\sum_{m \in I} \sum_{m^{\prime} \in I, m \neq m^{\prime}} K(n, m)^{*} K\left(n, m^{\prime}\right) \Gamma_{m}^{*} \Gamma_{m^{\prime}}
\end{gathered}
$$

The global Hilbert space interpretation is an ontological wave function, in that it describes the informons generated during one complete action of a process, and so one possible history of a quantum system. To carry out calculations, however, it is necessary to consider all possible histories. To do so requires the use of the process graph defined in the next section. It essentially is a combinatorial tool which keeps track of every possible history of the system as it evolves under a process from a fixed prior causal tapestry. Each possible history yields a distinct global Hilbert space interpretation. The Process Covering Map $\mathcal{P}(I)$ gathers together these interpretations into a single set valued map. From this one can form a combinatorial interpretation which can be used for calculations involving single systems. When multiple systems are involved, the process graph must be extended into a configuration space graph together with its associated configuration space covering map [2-4]. The details can be found elsewhere and are not needed for the arguments to follow.

It can be shown [2-4] that for a primitive process $\mathbf{P}$ and prior causal tapestry I, in the asymptotic limit as Planck length and Planck time tend to $0 ; r, N \rightarrow \infty, \mathcal{P}(I)\{\Psi(\mathbf{r})\}$, tends to a single function. Thus, in the case of a primitive process, in the asymptotic limit, the process generates only a single wave function which corresponds to the usual NRQM wave function. For a primitive process the wave function becomes both ontological and computational. This is not true for compound processes, so that the ontological wave function (global Hilbert space interpretation) which describes a single instance of reality, and the computational wave function which is used for making predictions, are no longer the same [24]. This failure to distinguish between these cases may be the source of much confusion about the interpretation of the wave function.

The process covering map gives rise to a correspondence between processes and (set-valued) operators on the space of global Hilbert space interpretations. The standard operator formalism is thus an emergent feature of the Process Algebra model arising in the asymptotic limit of infinite information and infinitesimal scale $[2,4,90]$. 
An important concept is that of epistemological equivalence. Epistemological equivalence of two processes $\mathbf{P}$ and $\mathbf{Q}$ means that their global Hilbert space interpretations, $\Psi^{P}(\mathbf{r}), \Psi^{Q}(\mathbf{r})$, respectively, are equal as functions over the causal manifold. In other words,

$$
\Psi^{P}(\mathbf{r})=\sum_{n} \Gamma_{n} f_{n}\left(\mathbf{r}, \mathbf{m}_{n}\right)=\sum_{m} \Gamma_{m} f_{m}\left(\mathbf{r}, \mathbf{m}_{m}\right)=\Psi^{Q}(\mathbf{r}) .
$$

If two processes are epistemologically equivalent then the specifics of informon generation do not matter in so far as NRQM is concerned. They generate the same emergent wave functions and therefore will yield the same NRQM predictions. This is useful because processes can be modeled heuristically based upon mathematical convenience just so long as they are epistemologically equivalent to any real processes. In particular one can use processes based upon combinatorial games which have particularly valuable characteristics [131133]. Epistemological equivalence may also possess ontological implications in that it might be impossible on principle for macroscopic observers to be able to access information about this most fundamental level. To use a computer analogy, it is generally inadvisable for a computer program to be able to access and change its own code. Perhaps that is the case for nature as well.

\section{THE PROCESS ALGEBRA}

The various paradoxes and conundrums posed by NRQM can be addressed through the Process Algebra. Processes can interact in a myriad of ways and the Process Algebra provides the formal language for describing these interactions. The power of epistemological equivalence is that it allows for many different representations of process to be considered based on heuristic, computational, or conceptual reasons, and it ensures that the results of calculations will still agree with one another. In this it is akin to the concept of gauge invariance. The most useful such representation to date is based upon combinatorial game theory. These games have been used for decades for generating mathematical structures [130-132] and are used heuristically as a model for how processes generate informons.

Processes may influence one another in two different ways. The first (coupling) involves the generation of individual informons, their relative timing as well as the sources of information which enters into their generation. Coupling results in epistemologically equivalent processes, so properties are unaltered. The second (interaction) involves the activation or inactivation of individual processes and the creation of new processes. Epistemological equivalence is broken and properties are altered.

Two processes $\mathbf{P}_{1}, \mathbf{P}_{2}$ may be independent, meaning that the neither constrains the actions of the other in any way. This relationship is denoted simply by the comma ",". Compound processes $(R>1)$ can be formed from primitive processes $(R$ $=1$ ) by various coupling operations. A coupling affects timing and information flow. Two processes may generate informons concurrently (products) during each round, or sequentially (sums), with only one process generating informons during a given round. Information from either or both processes may enter into the generation of a given informon (free) or information incorporated into an informon by a process may only come from informons previously generated by that process (exclusive). This leads to four possible operators:

1. Free sequential (free sum): $\mathbf{P}_{1} \hat{\oplus} \mathbf{P}_{2}$

2. Exclusive sequential (exclusive sum): $\mathbf{P}_{1} \oplus \mathbf{P}_{2}$

3. Free concurrent (free product): $\mathbf{P}_{1} \hat{\otimes} \mathbf{P}_{2}$

4. Exclusive concurrent (exclusive product): $\mathbf{P}_{1} \otimes \mathbf{P}_{2}$

The operation of concatenation is used to denote processes that act in successive generation cycles. Thus, $\mathbf{P}_{1} \cdot \mathbf{P}_{2}$ (or simply $\mathbf{P}_{1} \mathbf{P}_{2}$ ) indicates that $\mathbf{P}_{1}$ acts during the first generation cycle, while $\mathbf{P}_{2}$ acts during the second generation cycle.

Interactions break epistemological equivalence and can do so in myriad ways. Interactions between processes may activate an inactive process or inactivate an active process. In addition, an interaction among processes $\mathbf{P}_{1}, \mathbf{P}_{2}, . ., \mathbf{P}_{n}$ may generate a new process, $\mathbf{P}$, which can be described in functional form as $\mathrm{F}\left(\mathbf{P}_{1}, \mathbf{P}_{2}, \ldots, \mathbf{P}_{\mathrm{n}}\right)=\mathbf{P}$. If $\Theta\left(\mathbf{P}_{1}, \mathbf{P}_{2}, \ldots, \mathbf{P}_{\mathrm{n}}\right)$ describes a coupling among $\mathbf{P}_{1}, \mathbf{P}_{2}, \ldots, \mathbf{P}_{n}$ then the functional relation may be described using the operation of concatenation, as $\Theta\left(\mathbf{P}_{1}, \mathbf{P}_{2}, . ., \mathbf{P}_{n}\right) \mathbf{P}$.

Since there are potentially so many different types of interactions, a set of generic operators are used to indicate the presence of an interaction with the specifics to be spelled out if known. Thus, there are

1. Free sequential (free interactive sum): $\mathbf{P}_{1} \hat{\boxplus} \mathbf{P}_{2}$

2. Exclusive sequential (exclusive interactive sum): $\mathbf{P}_{1} \boxplus \mathbf{P}_{2}$

3. Free concurrent (free interactive product): $\mathbf{P}_{1} \hat{\otimes} \mathbf{P}_{2}$

4. Exclusive concurrent (exclusive product): $\mathbf{P}_{1} \otimes \mathbf{P}_{2}$

Independence, sums and products are commutative, associative and distributive operations. Concatenation is non-commutative and non-associative in general. The zero process, $\mathbf{O}$, is the process that does nothing.

An important and special form of interaction is the coupling interaction. Such interactions respect epistemological equivalence and thus are potentially reversible through a subsequent coupling interaction. An example is a rotation to a different eigenbasis as a result of an engagement with a measurement apparatus.

If the propagator is spatio-temporal invariant, so is the associated process. Since processes are independent of spacetime, their actions too are independent of any extrinsic causal manifold interpretation. They will act in the same manner regardless of where the embeddings into the causal manifold occur. Thus, if the propagator is invariant under space and time translations, so is the associated process.

Another point worth mentioning is that due to the noncommutativity of concatenation generally, there is an intrinsic temporal asymmetry within the process algebra model. Temporal evolution according to the process algebra model is not time reversible. It is quite permissible for two processes $\mathbf{P}, \mathbf{Q}$ to be time reversible individually, but yet their concatenation is not time reversible. Assume that $\mathbf{Q P} \neq \mathbf{P Q}$. If $T$ is the time reversal operator (which means that if $\mathbf{P}$ assigns an informon 
the causal manifold interpretation $(t, \mathbf{z})$, then $T[\mathbf{P}]$ assigns it the interpretation $(-t, \mathbf{z}))$, then $T[\mathbf{P}]=\mathbf{P}$ and $T[\mathbf{Q}]=\mathbf{Q}$ but $T[\mathbf{P Q}]=T[\mathbf{Q}] T[\mathbf{P}]=\mathbf{Q P} \neq \mathbf{P Q}$.

Unlike NRQM where multi-particle systems require tensor products, and QFT uses the Fock space, the multi-particle representation of the Process Algebra requires the use of a categorical co-product space. This space consists of formal, rather than arithmetic, sums of global Hilbert space interpretations. Thus, process algebra sums correspond to arithmetic sums while products correspond to co-product (formal) sums. Tensor products appear in the configuration space covering map, which again highlights the difference between the ontological global Hilbert space interpretation and the epistemological process and configuration space covering maps.

The impact of these different operations is best demonstrated using a process graph. The process graph $G(\mathbf{P})$ of a process $\mathbf{P}$ is defined as follows: rounds 0 to $\mathrm{N}$ are laid out in order. At round 0 one places the informons of the prior causal tapestry. At round $\mathrm{k}$, place each informon $\mathrm{n}$ that was generated during round $\mathrm{k}$ and draw a directed line from each prior informon in its content set $G_{\mathrm{n}}$ to $\mathrm{n}$ and label it withthe causal distance between the two informons. Note that no lines link informons of the prior causal tapestry to one another or nascent informons to one another since no information passes among them. Let $G(\mathbf{P}) n=\{n\} \cup G_{n}$, the subgraph of $G(\mathbf{P})$ consisting of $\mathrm{n}$ and its content set. The process graph is used to determine the causal manifold interpretation of the nascent causal tapestry and the global Hilbert space interpretation. If a process acts on the same prior causal tapestry it may produce a different process graph, thus a different history. The process covering map gathers together the global Hilbert space interpretations of these different process graphs, thus all of the possible histories required for a sum over histories calculation. A configuration space graph and configuration space covering map can be defined for products of processes.

Let $|\mathbf{P}|$ denote the total number of informons generated during the current generation cycle. For any two processes $\mathbf{P}, \mathbf{Q}$ we have

$$
\begin{gathered}
|\mathrm{P}|=N_{P},|\mathrm{Q}|=N_{\mathrm{Q}} \\
|\mathrm{P} \hat{\oplus} \mathrm{Q}|=|\mathrm{P} \oplus \mathrm{Q}|=\max \{|\mathrm{P}|,|\mathrm{Q}|\} \\
|\mathrm{P}, \mathrm{Q}|=|\mathrm{P} \hat{\otimes} \mathrm{Q}|=|\mathrm{P} \otimes \mathrm{Q}|=|\mathrm{P}|+|\mathrm{Q}| \\
|\mathrm{P} \cdot \mathrm{Q}|=|\mathrm{Q}|
\end{gathered}
$$

In addition we have

$$
G(P, Q)=G(P) \cup G(Q)
$$

$$
\begin{gathered}
G(\mathrm{P}) \cup G(\mathrm{Q})=G(\mathrm{P} \oplus \mathrm{Q}) \subset G(\mathrm{P} \hat{\oplus} \mathrm{Q}) \\
G(\mathrm{P}) \times G(\mathrm{Q})=G(\mathrm{P} \otimes \mathrm{Q}) \subset G(\mathrm{P} \hat{\otimes} \mathrm{Q}) \\
G(\mathrm{P} \cdot \mathrm{Q})=G(\mathrm{Q})
\end{gathered}
$$

This highlights some of the subtle differences between these operations.

The basic rules for applying these operations in combining processes are the following:

1 The free sum is only used for single systems and combining states which possess identical property sets (pure states).

2 The exclusive sum is used for single systems and combining states which possess distinct property sets (mixed states).

3 The free product is used for multiple systems which possess distinct character (scalar, spinorial, vectorial, and so on) such as coupling a boson and a fermion. It is unclear whether two bosons might couple via a free product.

4 The exclusive product is used for multiple systems which possess the same character such as coupling two bosons or two fermions.

The Process Algebra can be represented in many different ways as an algebra of processes, as an algebra of combinatorial games in one model of process, as an algebra of causal tapestry realizations, as a Hilbert space of global Hilbert space representations. Note that the latter representation is not faithful, that is it does not possess all of the structure of the Process Algebra. As stated previously, this results in a loss of causally meaningful information. To emphasize these different representations, one can describe Process Algebra elements as $|\mathbf{P}>,| G_{P}>$, $\left|\Omega_{P}(n)>,\right| \Psi_{P}(\mathbf{r})>$.

\section{CALCULATIONS IN THE PROCESS ALGEBRA MODEL}

To illustrate the difference in Hilbert space and Process Algebra approaches, consider first how the process algebra approach deals with superpositions. The linearity of the Schrödinger equation allows for two solutions $\Psi_{1}(\mathbf{r}), \Psi_{2}(\mathbf{r})$ to be summed together to yield a new solution $\Psi(\mathbf{r})=\alpha_{1} \Psi_{1}(\mathbf{r})+\alpha_{2} \Psi_{2}(\mathbf{r})$ of the same equation, and therefore a possible state. In the Hilbert space of NRQM note that every space-time point $\mathbf{r}$ possesses wave function contributions from both states $\Psi_{1}(\mathbf{r}), \Psi_{2}(\mathbf{r})$ and so if interpreted ontologically this means that the system at the point $\mathbf{r}$ is manifesting both states simultaneously, regardless of what those states entail. This is not a problem if the wave function is interpreted epistemologically (statistically) since in that case it is merely a tool to calculate the probability of being observed in either of the two states at the point r. This works for an ensemble of particles, but what of a single particle? How does one explain fixed probabilities if, unlike in classical probability, the object being observed 
possesses no definite attributes until after being observed? How is it that every observer determines the same attributes and probabilities?

By contrast consider the process algebra approach. Each component state $\Psi_{1}(\mathbf{r}), \Psi_{2}(\mathbf{r})$ is generated by its own process $\mathbf{P}_{1}, \mathbf{P}_{2}$, respectively. The superposition process is represented in the Process Algebra by the exclusive sum, $\mathbf{P}=\alpha_{1} \mathbf{P}_{1} \oplus \alpha_{2} \mathbf{P}_{2}$ so that each sub-process generates a unique, distinct causal tapestry, $I_{1}, I_{2}\left(I_{1} \cap I_{2}=\varnothing\right)$, respectively, and no information from one process enters into the generation of any informon of the other process. The two causal tapestries embed into disjoint regions of the causal manifold. The causal tapestries are thus physically and informationally isolated from one another. The causal tapestry for $\mathbf{P}$ is $I=I_{1} \cup I_{2}$ and on this causal tapestry the causal tapestry wave function $\Omega(n)$ for $\mathbf{P}$ takes the form $\Omega_{1}(n)+\Omega_{2}(n)$, where each $\Omega_{i}(n)$ is extended to $I$ by setting $\Omega_{i}(n)=0$ for $n \in I / I_{i}$, Note that although the algebraic sum is used there is no ontological confusion since for any $n, \Omega(n)$ receives contributions from either $\Omega_{1}(n)$ or $\Omega_{2}(n)$ but never both. Note though that the co-product free sum could also be used. This causal tapestry wave function describes a single system which manifests in either of the two states, which remain distinct, yet whose wave functions are intertwined. The informons that support these causal tapestry wave functions are generated sequentially, never concurrently, and so at any given moment only one of the two states is manifesting, so it only ever appears in a single state. The intertwining of the wave functions creates the impression of a mixed reality state at the macro-level, yet that is never the case at the micro-level.

In a superposition of processes, $\mathbf{P}=\alpha_{1} \mathbf{P}_{1} \oplus \alpha_{2} \mathbf{P}_{2}$ the effect of each modifier $\alpha_{i}$ is to modify the value of the local coupling effectiveness ( $\Gamma_{n} \rightarrow \alpha_{i} \Gamma_{n}$ for example) so that $\Omega(n)=$ $\alpha_{1} \Omega_{1}(n)+\alpha_{2} \Omega_{2}(n)$ and the global Hilbert space interpretation is formed in the usual manner, $\Psi(\mathbf{r})=\alpha_{1} \Psi_{1}(\mathbf{r})+\alpha_{2} \Psi_{2}(\mathbf{r})$.

The combined global Hilbert space interpretation thus takes the form

$$
\begin{array}{r}
\Psi(\mathbf{r})=\sum_{n \in I_{1} \cup I_{2}} \Gamma_{n} f_{n}\left(\mathbf{r}, \mathbf{m}_{n}\right)=\sum_{n \in I_{1}} \alpha_{1} \Gamma_{n} f_{n}\left(\mathbf{r}, \mathbf{m}_{n}\right) \\
+\sum_{n \in I_{2}} \alpha_{2} \Gamma_{n} f_{n}\left(\mathbf{r}, \mathbf{m}_{n}\right)=\alpha_{1} \Psi_{1}(\mathbf{r})+\alpha_{2} \Psi_{2}(\mathbf{r}),
\end{array}
$$

This is a map in the Hilbert space $H(\boldsymbol{M})$ over the causal manifold, the same Hilbert space where the NRQM wave function resides, and it constitutes the Process Algebra approximation to the NRQM wave function. Note that in moving to the Hilbert space, causal ontological information is lost since now the wave function is over space-time locations, not informons, and contributions from the sub-processes are now summed, not intertwined. This information could be preserved if the algebraic sum above were replaced by a co-product free sum, but that is not a Hilbert space property. However, it would not be possible to carry out the usual NRQM calculations in that case.

The concept of informon being new, there is currently no direct evidence to suggest a particular model ofr their generation. Epistemological equivalence, however, allows one to side step that for the moment, and any strategy resulting in an epistemologically equivalent model suffices. Several such models have been presented in the literature $[2-6,90]$. Work is underway to model informon generation as a diffusion process as has been suggested in the literature for the Schrödinger equation $[111,114,128]$. The discussion here is meant as an in-principle demonstration of the Process Algebra framework and not a final theory.

The causal tapestry wave function is ontological, representing a single complete action of a process in generating informons (and thus a region of space-time events). The causal tapestry wave function represents the outcome of interactions with other processes only with respect to that single process. Statistical calculations require the use of the process and configuration space graphs and covering maps. The compatibility between processes is conjectured to be a function of the local coupling effectiveness $\Gamma_{n}$, which in turn reflects the effect of myriad local interactions. If the effect of these interactions is summarized in a potential, then it seems reasonable, as an initial approximation, to assume that the local coupling effectiveness will depend in some manner upon the Lagrangian. The probability of an interaction, triggered by the generation of informons, must be positive real valued and is conjectured to depend on the compatibility. It seems reasonable, therefore, to conjecture that the compatibility (hence probability) should depend on the local process strength, $\Gamma_{n}^{*} \Gamma_{n}$ which is both positive real and relativistically invariant. Therefore, making the simplest assumption, the local probability is assumed to be given by the Born rule, $P_{n}=\Gamma_{n}^{*} \Gamma_{n}$. If an interaction depends upon the presence of several informons $A=\left\{n_{i}\right\}$, then the probability depends upon $P_{A}=\sum_{m \in A} \Gamma_{n}^{*} \Gamma_{n}$, the local process strength over $A$. As in NRQM these local coupling effectiveness values can be normalized relative to the global process strength $P_{I}=\sum_{n \in I} \Gamma_{n}^{*} \Gamma_{n}=|\Omega(n)|^{2}$. Recall from a previous section that interaction effects are already encoded within the $\Gamma_{n}$. By analogy with Dirac's bra-ket formalism, one can introduce a scalar product on the causal tapestry wave function of the form $<\Omega(n) \mid \Omega^{\prime}(m)>=\sum_{n, m \in I} \Omega^{*}(n) \Omega^{\prime}(m) \delta(n, m)$ where $\delta(n, m)$ is akin to a differential. The percentage of contribution to the strength by the process generating $\Omega(n)$ to the process generating $\Omega^{\prime}(m)$ is given by $|<\Omega(n)| \Omega^{\prime}(m)>\left.\right|^{2}$. The global strength is given as $|\Omega(n)|^{2}=\sum_{n \in I} \sum_{m \in I} \Omega^{*}(n) \Omega(m) \delta(n, m)=$ $\sum_{n \in I} \Gamma_{n}^{*} \Gamma_{n}$.

For example if $\Omega(n)=\alpha_{1} \Omega_{1}(n)+\alpha_{2} \Omega_{2}(n)$ then $<\Omega_{2}(n) \mid \Omega(n)>=\sum_{n \in I_{2}} \sum_{m \in I_{1} \cup I_{2}} \Omega_{2}^{*}(n) \Omega(n) \delta(n, m)=\alpha_{2} \sum_{n \in I_{2}}$ $\Gamma_{n}^{*} \Gamma_{n}=\alpha_{2}\left|\Omega_{2}(n)\right|^{2}=\alpha_{2}$

assuming suitable normalization of the process strength

These calculations can also be carried out using the global Hilbert space interpretation with the usual Hilbert space scalar product $<\Psi(\mathbf{r}) \mid \Psi^{\prime}(\mathbf{r})>=\int_{M} \Psi^{*}(\mathbf{r}) \Psi^{\prime}(\mathbf{r}) d \mathbf{r}$.

Conisder a region $\hat{A}$ of the causal manifold containing causal manifold interpretations of informons in the set $A$. Then we may define 


$$
\begin{aligned}
P_{\hat{A}}=\int_{\hat{A}} \Psi^{*}(\mathbf{r}) \Psi(\mathbf{r}) d \mathbf{r}=\int_{\hat{A}} \sum_{n, n^{\prime} \in I} \Gamma_{n}^{*} \Gamma_{n^{\prime}} f_{n}\left(\mathbf{r}, \mathbf{m}_{\mathbf{n}}\right) f_{n^{\prime}}\left(\mathbf{r}, \mathbf{m}_{\mathbf{n}^{\prime}}\right) d \mathbf{r}= \\
\quad \int_{\hat{A}} \sum_{n, n^{\prime} \in A} \Gamma_{n}^{*} \Gamma_{n^{\prime}} f_{n}\left(\mathbf{r}, \mathbf{m}_{\mathbf{n}}\right) f_{n^{\prime}}\left(\mathbf{r}, \mathbf{m}_{\mathbf{n}^{\prime}}\right) d \mathbf{r} \\
+\int_{\hat{A}} \sum_{n, n^{\prime} \notin A} \Gamma_{n}^{*} \Gamma_{n^{\prime}} f_{n}\left(\mathbf{r}, \mathbf{m}_{\mathbf{n}}\right) f_{n^{\prime}}\left(\mathbf{r}, \mathbf{m}_{\mathbf{n}^{\prime}}\right) d \mathbf{r} \approx \\
\\
\quad \int_{\hat{A}} \sum_{n, n^{\prime} \in A} \Gamma_{n}^{*} \Gamma_{n^{\prime}} f_{n}\left(\mathbf{r}, \mathbf{m}_{\mathbf{n}}\right) f_{n^{\prime}}\left(\mathbf{r}, \mathbf{m}_{\mathbf{n}^{\prime}}\right) d \mathbf{r}
\end{aligned}
$$

since the $f_{n}$ decrease in value rapidly away from $A$. Similarly,

$$
\begin{aligned}
& \int_{M} \sum_{n, n^{\prime} \in A} \Gamma_{n}^{*} \Gamma_{n^{\prime}} f_{n}\left(\mathbf{r}, \mathbf{m}_{\mathbf{n}}\right) f_{n^{\prime}}\left(\mathbf{r}, \mathbf{m}_{\mathbf{n}^{\prime}}\right) d \mathbf{r}= \\
& \int_{A} \sum_{n, n^{\prime} \in A} \Gamma_{n}^{*} \Gamma_{n^{\prime}} f_{n}\left(\mathbf{r}, \mathbf{m}_{\mathbf{n}}\right) f_{n^{\prime}}\left(\mathbf{r}, \mathbf{m}_{\mathbf{n}^{\prime}}\right) d \mathbf{r} \\
& +\int_{M / A} \sum_{n, n^{\prime} \in A} \Gamma_{n}^{*} \Gamma_{n^{\prime}} f_{n}\left(\mathbf{r}, \mathbf{m}_{\mathbf{n}}\right) f_{n^{\prime}}\left(\mathbf{r}, \mathbf{m}_{\mathbf{n}^{\prime}}\right) d \mathbf{r} \approx \\
& \int_{A} \sum_{n, n^{\prime} \in A} \Gamma_{n}^{*} \Gamma_{n^{\prime}} f_{n}\left(\mathbf{r}, \mathbf{m}_{\mathbf{n}}\right) f_{n^{\prime}}\left(\mathbf{r}, \mathbf{m}_{\mathbf{n}^{\prime}}\right) d \mathbf{r} .
\end{aligned}
$$

Thus

$$
\begin{aligned}
& \int_{\hat{A}} \sum_{n, n^{\prime} \in I} \Gamma_{n}^{*} \Gamma_{n^{\prime}} f_{n}\left(\mathbf{r}, \mathbf{m}_{\mathbf{n}}\right) f_{n^{\prime}}\left(\mathbf{r}, \mathbf{m}_{\mathbf{n}^{\prime}}\right) d \mathbf{r} \approx \\
& \int_{M} \sum_{n, n^{\prime} \in A} \Gamma_{n}^{*} \Gamma_{n^{\prime}} f_{n}\left(\mathbf{r}, \mathbf{m}_{\mathbf{n}}\right) f_{n^{\prime}}\left(\mathbf{r}, \mathbf{m}_{\mathbf{n}^{\prime}}\right) d \mathbf{r}=\sum_{n \in A} \Gamma_{n}^{*} \Gamma_{n}
\end{aligned}
$$

again since the $f_{n}$ are orthogonal to one another. Likewise

$$
\begin{aligned}
& <\Psi_{2}(\mathbf{r}) \mid \Psi(\mathbf{r})>=\int_{\mathbf{r}} \sum_{n \in I_{2}} \Gamma_{n}^{*} f_{n}\left(\mathbf{r}, \mathbf{m}_{n}\right)\left(\sum_{m \in I_{1}} \alpha_{1} \Gamma_{m} f_{m}\left(\mathbf{r}, \mathbf{m}_{m}\right)\right. \\
& \left.+\sum_{m^{\prime} \in I_{2}} \alpha_{2} \Gamma_{m^{\prime}} f_{m^{\prime}}\left(\mathbf{r}, \mathbf{m}_{m^{\prime}}\right)\right) d \mathbf{r}=\alpha_{2} \sum_{n \in I_{2}} \Gamma_{n}^{*} \Gamma_{n}=\alpha_{2}
\end{aligned}
$$

Thus, the basic calculations can all be carried out on the causal tapestry itself and so as stated previously, all of the relevant physics occurs on the causal tapestry. The global Hilbert space interpretation provides an observer dependent link to the NRQM Hilbert space which facilitates some calculations and comparisons to NRQM but is not necessary for the physics.
Each causal tapestry wave function $\Omega(n)$ provides an ontological representation of the action of a single process, and thus the history of one occurrence of that process. This is insufficient to carry out computations because only $N$ informons will be generated out of a possible infinitude. The causal tapestry wave function may suffice for primitive processes under certain asymptotic conditions, but it fails for compound processes as it generates only $\mathrm{N}$ informon tuples whereas at least $\mathrm{N}^{\mathrm{R}}$ must be determined. For computations one must resort to the process covering graph. There all possible causal tapestry histories are gathered (by union) into a single causal graph and the causal tapestry wave function is generated on this graph, as is the global Hilbert space interpretation. These maps now contain sufficient information about all possible evolutions to make calculations possible. However, the process graph and its wave functions are not ontological; they are merely epistemological structures used to carry out calculations.

The information incorporated into the local coupling effectiveness (and local process strength) takes into account local effects both of and upon the generating process. In the course of generating informons it is presumed that there will be interactions with other processes. When these other processes give rise to relatively persistent macroscopic or classical-like entities it is convenient to summarize these local effects in terms of field notions such as the potential field. The local coupling coefficients should take these effects into account so a natural first choice is to try a Lagrangian approach.

Assume now that a suitable strategy for generating informons has been adopted and that the informons are generated so as to form points on a $4 \mathrm{D}$ lattice with lattice spacing $\left(l_{P}\right)^{4}$ where $l_{P}$ is the Planck length. For simplicity, assume further that one has a primitive process $(R=1)$ in some energy eigenstate (so we need only consider a single causal tapestry) and that $N=r=|c|$, the value of $c$ to the nearest integer and stripped of its units. Assume that each generation of an informon occurs in Planck time $t_{P}$ and that each complete action of the process generates $|c|$ informons in time $|c| t_{P}$. This time interval corresponds to a length $c t_{P}=l_{P}$. Thus, each causal tapestry is separated from the next by an interval $c t_{P}=l_{P}$, hence the choice of lattice spacing. Label each causal tapestry by its generation number $n$ so that the time coordinate for the $n$th tapestry is $\mathrm{n}|c| t_{P}$ and each causal tapestry corresponds to a $3 \mathrm{D}$ spatial lattice.

Initial states are generally assumed in NRQM. In the Process Algebra framework, since causal tapestries are generated, so must initial states, and so the question of initial states is actually rather important and subtle within the Process Algebra framework. However, a discussion of this problem would detract from the main focus and an initial causal tapestry $I_{0}$ will simply be assumed with causal tapestry wave function $\Omega_{0}(n)$, global Hilbert space interpretation $\Psi_{0}(\mathbf{r})$ and corresponding NRQM wave function $\hat{\Psi}_{0}(\mathbf{r})$ satisfying $\Psi_{0}\left(\mathbf{m}_{n}\right)=\hat{\Psi}_{0}\left(\mathbf{m}_{n}\right)$ on the embedding sites of informons. Each subsequent causal tapestry is labeled by its generation number $n$ and denote the corresponding $3 \mathrm{D}$ sublattice as $L_{n}$.

An effective space-time approach is due to Feynman [129]. However, this is presented here only to provide an "in-principle" demonstration. The Feynman propagator allows for long range 
transfer of information (long range "paths") and is suitable for NRQM where causal has a different definition but it is not causal in the relativistic sense. Restricting the number of informons being generated serves to truncate the calculation and avoid this problem but then to be precise more definite estimates of the Kernel approximation are needed to determine the size of the resulting amplitude error. These details warrant another paper. If the Lagrangian for a scalar particle of mass $m$ is $\mathcal{L}=p^{2} / 2 m-$ $V$ then on the causal tapestry one might expect it to take the form $\mathcal{L}\left(n, n^{\prime}\right)=m d\left(n, n^{\prime}\right)^{2} / 2|c|^{2} t_{P}^{2}-V(n)$ where $n$ and $n^{\prime}$ refer to informons on the nascent and current tapestries, respectively, and $d$ is the causal tapestry distance. Then $S\left[n, n^{\prime}\right]=\mathcal{L}\left(n, n^{\prime}\right)|c| t_{P}$ so the propagator may be written as $\mathcal{P}_{n, n^{\prime}}=\frac{l_{P}^{3}}{A^{3}} e^{i / \hbar S\left[n, n^{\prime}\right]}$ Note that this is defined entirely on the causal tapestry.

The tapestry wave function can be extended from the tapestry $I_{n}$ to the sublattice $L_{n}$ by the convention that $\Omega_{m}(n)=0$ if $n \notin I_{n}$ and that $\mathcal{P}_{n, n^{\prime}}=0$ if no information propagates from $n$ to $n$ '. Assume that the process has generated tapestries up to generation $m$ and consider generation $m+1$. Assume that $N$ informons have been generated and consider the causal tapestry wave function $\Omega_{m+1}(n)$. The value of the local coupling effectiveness at the informon $n^{m+1}$ is $\Gamma^{m+1}{ }_{n^{m+1}}=\sum_{n^{m} \in I_{m}} \mathcal{P}_{n^{m+1}, n^{m}} \Gamma^{m} n^{m}$. Expanding back to the initial state one has

$$
\begin{gathered}
\Gamma_{n^{m+1}}^{m+1}=\sum_{n^{m} \in \mathcal{L}_{m}} \cdots \sum_{n^{0} \in \mathcal{L}_{0}} \mathcal{P}_{n^{m+1} n^{m}} \cdots \mathcal{P}_{n^{1} n^{0}} \Gamma_{n 0}^{0}= \\
\Gamma_{n^{m+1}}^{m+1}=\sum_{n^{m} \in \mathcal{L}_{m}} \cdots \sum_{n^{0} \in \mathcal{L}_{0}} \mathcal{P}_{n^{m+1} n^{m}} \cdots \mathcal{P}_{n^{1} n^{0}} \hat{\Psi}_{0}\left(\mathbf{m}_{n^{0}}\right)= \\
\sum_{n^{m} \in \mathcal{L}_{m}} \cdots \sum_{n^{0} \in \mathcal{L}_{0}} \frac{l_{P}^{3}}{A^{3}} e^{\frac{i}{\hbar} S\left[n^{m+1}, n^{m}\right]} \frac{l_{P}^{3}}{A^{3}} e^{\frac{i}{\hbar} S\left[n^{m}, n^{m-1}\right]} \cdots \times \frac{l_{P}^{3}}{A^{3}} e^{\frac{i}{\hbar} S\left[n^{1}, n^{0}\right]} \hat{\Psi}_{0}\left(\mathbf{m}_{n^{0}}\right)= \\
\sum_{n^{m} \in \mathcal{L}_{m}} \cdots \sum_{n^{0} \in \mathcal{L}_{0}} e^{\frac{i}{\hbar} S\left[n^{m+1}, n^{m}\right]+S\left[n^{m}, n^{m-1}\right]+\cdots+S\left[n^{1}, n^{0}\right]} \times \overbrace{\frac{l_{P}^{3}}{A^{3}} \frac{l_{P}^{3}}{A^{3}} \cdots \frac{l_{P}^{3}}{A^{3}}}^{\Psi_{0}} \hat{\Psi}_{0}\left(\mathbf{m}_{n^{0}}\right)=
\end{gathered}
$$

(using Feynman's notation)

$$
\begin{gathered}
\sum_{n^{m} \in \mathcal{L}_{m}} \cdots \sum_{n^{0} \in \mathcal{L}_{0}} e^{\frac{i}{h} S\left[n^{m+1}, n^{0}\right]} \overbrace{\frac{l_{P}^{3}}{A^{3}} \frac{l_{P}^{3}}{A^{3}} \cdots \frac{l_{P}^{3}}{A^{3}}}^{m+1} \hat{\Psi}_{0}\left(\mathbf{m}_{n^{0}}\right) \approx \\
\sum_{n^{0} \in \mathcal{L}_{0} L_{m}} \cdots \int_{L_{1}} e^{\frac{i}{\hbar} S\left[n^{m+1}, n^{0}\right]} \overbrace{\frac{d x^{m+1}}{A^{3}} \cdots \frac{d x^{1}}{A^{3}}}^{m} \frac{l_{P}^{3}}{A^{3}} \hat{\Psi}_{0}\left(\mathbf{m}_{n^{0}}\right) \approx \\
\int_{L_{m}} \cdots \int_{L_{0}} e^{\frac{i}{\hbar} S\left[n^{m+1}, n^{0}\right]} \overbrace{\frac{d x^{m+1}}{A^{3}} \cdots \frac{d x^{0}}{A^{3}}}^{m+1} \hat{\Psi}_{0}(\mathbf{r})=\hat{\Psi}_{m+1}\left(\mathbf{m}_{n^{m+1}}\right)
\end{gathered}
$$

Thus, we find that the global Hilbert space interpretation

$$
\begin{gathered}
\Psi_{m+1}(\mathbf{r})=\sum_{n^{m+1} \in L_{m+1}} \Gamma_{n^{m+1}}^{m+1} f_{n^{m+1}}\left(\mathbf{r}, \mathbf{m}_{n^{m+1}}\right) \approx \\
\sum_{n^{m+1} \in L_{m+1}} \hat{\Psi}_{m+1}\left(\mathbf{m}_{n^{m+1}}\right) f_{n^{m+1}}\left(\mathbf{r}, \mathbf{m}_{n^{m+1}}\right) \approx \hat{\Psi}_{m+1}(\mathbf{r})
\end{gathered}
$$

The accuracy of the approximation will depend upon N,r and the value of $\frac{l}{p}$. Parzen's theorem [121] can be used to show that it will become increasingly exact as $N, r \rightarrow \infty$ and $l_{P} \rightarrow 0$. In the Process Algebra framework, the usual approximation of $h \rightarrow 0$ does not lead to the classical realm but merely to NRQM. This is the reason that NRQM is considered to be an effective theory in the limit of infinite information $N, r \rightarrow \infty$ and continuity or infinitesimal scale $h \rightarrow 0$ Classicality is thought to be a consequence of the complexity process interactions.

The causal tapestry wave function is discrete and finite and so clearly can diverge in values from the corresponding NRQM wave function. These differences can be due to a variety of factors: truncation errors due to the finite number of informon generated, aliasing errors due to the informon density, amplitude errors due to the discrete approaximation to the Kernel integral, time-jitter errors due to non-uniform spacing of informons and information loss if informons are generated non-contiguously [121]. However, if the density of informons exceeds the Beurling density for the NRQM wave function and if the wave function tends to zero at infinity rapidly, then the difference between these two may be surprisingly small. In the case of a primitive process generating a single eigenstate the NRQM wave function can be achieved in one of two ways-either or by the asymptotic procedure described above or by resorting to the process graph and covering map. Both techniques allow for information to divege to infinity, thus capturing all possibly informon generation sequences and therefore, information paths. The causal tapestry itself, however, is always discrete and finite and possesses finite information, which will result in a disagreement between the global Hilbert space interpretation and the NRQM wave function. This error will depend upon the accuracy of the approximation to the integral $\int_{\mathcal{M}} K\left(\mathbf{r}_{j^{\prime}}, \mathbf{r}_{j}\right) \phi_{j}\left(\mathbf{r}_{j}\right) d \mathbf{r}_{j}$, the deviations from uniformity of the informons, the values of $N, r$ and of $t_{P}, l_{P}$. Determining the error in the general case is probably intractable but there are results for special cases, particularly when the informons occupy contiguous sites in a regular lattice such as in the example above. In one dimension, if the NRQM wave function $\hat{\Psi}(t)$ satisfies $|\hat{\Psi}(t)| \leq M|t|^{-\gamma}$ for $0<\gamma \leq 1$, $\left|\int_{\mathcal{M}} K\left(\mathbf{r}_{j^{\prime}}, \mathbf{r}_{j}\right) \phi_{j}\left(\mathbf{r}_{j}\right) d \mathbf{r}_{j}-\Psi\left(\mathbf{r}_{j^{\prime}}\right)\right| \leq \epsilon$, the discrepancy between each embedding point and its ideal lattice embedding point is less than $\delta$, and the truncation number $r=2\left[W^{1+1 / \gamma}+\right.$ $1]+1$, then according to a theorem of Butzer [121], the error E satisfies

$$
\|E\|_{\infty} \leq-K\left(\Psi, \gamma, \epsilon / l_{P}, \delta / l_{P}\right) l_{P} \ln l_{P}
$$


where

$$
K=\left(1+\frac{1}{\gamma}\right)\left\{\sqrt{5} e\left[\left(\frac{14}{\pi}+\delta / l_{P}+\frac{7}{3 \sqrt{5} \pi}\right)\left\|\Psi^{(1)}\right\|_{\infty}+\grave{\mathrm{o}} / l_{P}\right]+6 e\left(M+\|\Psi\|_{\infty}\right)\right\}
$$

Then $\|E\|_{\infty} \approx 10^{-33} \mathrm{~K}$ if $l_{P}$ is the Planck length.

Ideally, if the discrete approximation to the Kernel integral and the Kernel integral are equal, the informons lie continguously on a uniform lattice, and the informons occupy a cubic spatial region with $N^{1 / 3}$ informons on one side, then one can use the Yao and Thomas theorem [121] to find a rough estimate for the error $E$, namely

$$
|\Phi(\mathbf{r})-\Psi(\mathbf{r})|=|E| \leq \frac{64 \max _{\mathcal{M}^{\prime}}|\Psi(x, y, z)|}{(2 \pi)^{3} N} \approx \frac{1}{31 N}\left(m^{-3 / 2}\right) .
$$

Thus, a value for $N$ of $|c|$ yields an error of $\sim 10^{-10}\left(\mathrm{~m}^{-3 / 2}\right)$ and if $N=|c|^{3}$ then the error is $\sim 10^{-30}\left(\mathrm{~m}^{-3 / 2}\right)$.

Thus, even this relatively simple Process Algebra model can reproduce, to a high degree of accuracy, any NRQM wave function which can be calculated using Feynman path integrals and whose energy is bounded by $\mathrm{h} / 2|\mathrm{c}| \mathrm{t}$.

\section{THE PROCESS ALGEBRA APPROACH TO THE PARADOXES}

The paradoxes and dualities of quantum mechanics appear to arise from one of two main factors, either a failure to utilize a contextual probability model when analyzing an experimental situation, or an attempt to interpret a wave function in both ontological and epistemological terms. The former was pointed out by Dzhafarov [60] in his contextuality by default analysis of the two slit experiment. The difference in outcomes is a consequence of contextuality. It can also be seen in the analysis of wave-particle duality by Ionicioiu et al. [18] who used a Bell type argument to try to show that the wave-particle distinction for a particle cannot be non-contextual.

The Process Algebra approach focuses upon the latter factor, the confusion of ontology and epistemology. Only the causal tapestry realization is truly ontological, although the global Hilbert space interpretation preserves ontology at informon embeddings sites. For epistemology one requires the use of the process and configuration space graphs and the setvalued process and configuration space covering maps. These lead to the usual NRQM wave functions, which are viewed as being epistemological. The resolution of the paradoxes is not to be had through an ever more clever or convoluted elaboration of standard quantum mechanics using the Hilbert space. It is not a problem of computation. The problem lies in attempting to use the Hilbert space formalism to provide an ontological model of quantum mechanics. The Hilbert space is simply too coarse grained to carefully distinguish between distinct ontological states. This coarse graining is adequate for carrying out calculations of quantum mechanical statistics, but not for the purpose of ontology. The problem is that the Hilbert space serves as a quotient space relative to the Process Algebra, which results in a loss of ontological information. The Hilbert space conflates information relative to distinct ontological states leading to confusion when attempts are made to provide an ontological interpretation. This does not occur in the Process Algebra framework. Thus, the problem is not with reality, but rather with the mathematical language used to represent reality.

\section{CONCLUSION}

Theoretical and experimental evidence strongly points to the end of local non-contextual hidden variables. This, however, is not the end of local realism. The fundamental result is that reality is contextual. This is entirely compatible with locality. There is no need to introduce non-locality or "spooky action at a distance." There need be no conflict between quantum mechanics and relativity. The cost, however, is to abandon the principle of continuity and accept a process generated reality. A specifc model, the Process Algebra model is presented as an in-principle demonstration of model which is generated, causally local, Lorentz invariant, contextual, without hidden variables and which. can reproduce the results of NRQM to as high level of accuracy. It is suggested that the paradoxes that plague quantum mechanics are due to the inability of the Hilbert space formalism to correctly distinguish between ontology and epistemology. The Process Algebra model corrects this deficiency and promises the possibility of a paradox free quantum mechanics. Hopefully this paper will encourage further research.

\section{DATA AVAILABILITY STATEMENT}

The original contributions presented in the study are included in the article/supplementary material, further inquiries can be directed to the corresponding author/s.

\section{AUTHOR CONTRIBUTIONS}

The sole author is responsible for the literature review, conceptualization, analysis, and preparation of the manuscript. Any errors and omissions are their sole responsibility.

\section{ACKNOWLEDGMENTS}

WS wishes to thank Irina Trofimova for countless discussions related to process ideas. WS would also like to thank Andrei Khrennikov and Etibar Dzhafarov for many valuable discussions. Further, WS wishes to thank Robb Mann, Rafael Sorkin and Achim Kempf for their support during the gestation of the early version of these ideas. WS also wishes to thank the reviewers who provided very helpful commentary and additional references which helped to improve the quality of the paper. 


\section{REFERENCES}

1. Einstein A, Podolsky B, Rosen N. Can quantum mechanical description of reality be considered complete? Phys Rev. (1935) 77:777-80. doi: 10.1103/PhysRev.47.777

2. Sulis W. A Process Model of Non-Relativistic Quantum Mechanics. Ph.D. thesis, University orf Waterloo, Waterloo, ON (2014).

3. Sulis W. A process model of quantum mechanics. J Mod Phys. (2014) 5: 1789-95. doi: 10.4236/jmp.2014.516176

4. Sulis W. Completing quantum mechanics. In: Sienicki K, editor. Quantum Mechanics Interpretations. Berlin: Open Academic Press (2017) p. 350-421.

5. Sulis W. A process algebra approach to quantum electrodynamics: physics from the top up. In: Martinez R, editor. Complex Systems: Theory and Applications. New York, NY: Nova Publishing (2017). p. 1-42.

6. Sulis W. A process algebra approach to quantum electrodynamics. Int J Theoret Phys. (2017) 56:3869-79. doi: 10.1007/s10773-017-3366-y

7. Colbeck R, Renner R. No extension of quantum theory can have improved predictive power. Nat. Comm. (2011) 2:411. doi: 10.1038/ncomms1416

8. Dawid R. String Theory and the Scientific Method. Cambridge: Cambridge University Press (2014). doi: 10.1017/CBO9781139342513

9. Bohm D. A suggested interpretation of the quantum theory in terms of "hidden variables". I. Phys Rev. (1952) 85:16679. doi: 10.1103/PhysRev.85.166

10. Bohm D, Aharonov Y. Discussion of experimental proof for the paradox of Einstein, Rosen, and Podolsky". Phys Rev. (1957) 108:1070. doi: 10.1103/PhysRev.108.1070

11. Bell JS. Speakable and Unspeakable in Quantum Mechanics. Cambridge: Cambridge University Press (1987).

12. Clauser J, Horne M, Shimony A, Holt R. Proposed experiment to test local hidden variable theories. Phys Rev Lett. (1969) 23:8804. doi: 10.1103/PhysRevLett.23.880

13. Shimony A. Search for a Naturalistic World View, Volume II, Natural Science and Metaphysics. Cambridge: Cambridge University Press (1993). doi: 10.1017/CBO9780511621147

14. Jarrett J. On the physical significance of the locality conditions in the Bell argument. Nous. (1984) 18:569-89. doi: 10.2307/2214878

15. Clauser J, Shimony A. Bell's theorem : experimental tests and implications. Rep Prog Phys. (1978) 41:1881-927. doi: 10.1088/0034-4885/41/12/002

16. Leggett AJ, Garg A. Quantum mechanics versus macroscopic realism: Is the flux there when nobody looks? Phys Rev Lett. (1985) 54:85760. doi: 10.1103/PhysRevLett.54.857

17. Bancal JD, Pironio S, Acin A, Liang YC, Scarani V, Gisin N. Quantum nonlocality based on finite-speed causal influences leads to superluminal signalling. Nature Phys. (2012) 8: 867-70. doi: 10.1038/nphys2460

18. Ionicioiu $\mathrm{R}$, Jennewein $\mathrm{T}$, Mann RB, Terno, D. Is wave-particle objectivity compatible with determinism and locality? Nat Comm. (2014) 5:3997. doi: 10.1038/ncomms5997

19. Fine, A. Hidden variables, joint probability, and the Bell inequalities. Phys Rev Lett. (1982) 48:291-5. doi: 10.1103/PhysRevLett.48.291

20. Fine A. Joint distributions, quantum correlations, and commuting observables. J Math Phys. (1982) 23:1306-10. doi: 10.1063/1.525514

21. Kolmogorov AN. Foundations of the Theory of Probability. New York, NY: Chelsea Publishing (1956).

22. Vorob'ev NN. Consistent families of measures and their extensions. Theory Prob Appl. (1962) 7:147-63. doi: 10.1137/1107014

23. Handsteiner J, Friedman A, Rauch. D, Gallicchio A, Liu B, Hosp H, et al. Cosmic Bell test: measurements settings from Milky Way stars. Phys Rev Lett. (2017) 118:060401. doi: 10.1103/PhysRevLett.118.060401

24. von Neumann, J. Mathematical Foundations of Quantum Mechanics. Princeton: Princeton University Press (1955).

25. Gleason AM. Measures on the closed subspaces of a Hilbert space. J Math Mech. (1957) 6:885-93. doi: 10.1512/iumj.1957.6.56050

26. Mackey G. Quantum mechanics and Hilbert space. Am Math Monthly. (1957) 64:45-57. doi: 10.1080/00029890.1957.11989120

27. Kochen S, Specker E. The problem of hidden variables in quantum mechanics. J Math Mech. (1967) 17:59-87. doi: 10.1512/iumj.1968.17.17004

28. Mermin D. Simple unified form for the major no-hidden-variables theorem. Phys Rev Lett. (1990) 65:3373-6. doi: 10.1103/PhysRevLett.65.3373
29. Wheeler JA, ZurekWH. Quantum theory and measurement. Princeton: Princeton University Press (1983).

30. Aspect A. Closing the door on Einstein's and Bohr's quantum debate. Physics. (2015) 8:123-6. doi: 10.1103/Physics.8.123

31. Hensen B, Bernien H, Dréau AE, Reiserer A, Kalb N, Blok MS, et al. Loophole-free Bell inequality violation using electron spins separated by 1.3 kilometres. Nature. (2015) 526:682-6. doi: 10.1038/nature15759

32. Shalam L, Meyer-Scott E, Christensen B, Bierhorst P, Wayne M, Stevens M, et al. Strong loop-hole free test of local realism. Phys Rev Lett. (2015) 115:250402. doi: 10.1103/PhysRevLett.115.250402

33. Giustina M, Versteegh M, Wengerowsky S, Handsteiner J, Hochrainer A, Phelan K, et al. Significant-loophole-free test of Bell's theorem with entangled photons. Phys Rev Lett. (2015) 115:250401. doi: 10.1103/PhysRevLett.115.250401

34. Hensen B, Kalb N, Blok MS, Dréau EA, Reiserer A, Vermeulen RFL, et al. Loophole-free Bell test using electron spins in diamond: second experiment and additional analysis. Sci Rep. (2016) 6:30289. doi: 10.1038/srep30289

35. Rauch D, Handsteiner J, Hochrainer A, Gallicchio J, Friedman A, Leung C, et al. Cosmic Bell test using random measurement settings from high red-shift quasars. Phys Rev Lett. (2018) 121:080403. doi: 10.1103/PhysRevLett.121.080403

36. Proietti M, Pickston A, Graffitti F, Barrow P, Kundys D, Branciard C, et al. Experimental test of local observer-independence. arXiv [Preprint]. (2019). arXiv: 1902. 05080v2. doi: 10.1126/sciadv.aaw9832

37. Dhand I, D'Souza A, Narasimhachar V, Sinclair N, Wein S, Zarkeshian P, et al. Understanding quantum physics through simple experiments: from wave-particle duality to Bell's theorem. arXiv [Preprint]. (2018).

38. Moreau P-A, Toninelli E, Gregory T, Aspden R, Morris P, Padgett M. Imaging Bell-type nonlocal behavior. Sci Adv. (2019) 5:eaaw2563. doi: 10.1126/sciadv.aaw2563

39. Conway JH, Kochen S. The free will theorem. arXiv. (2006). doi: 10.1007/s 10701-006-9068-6

40. Khrennikov A. Getting rid of non-locality from quantum physics. arXiv [Preprint]. (2019). arXiv: 1907 02702v3. doi: 10.20944/preprints202004.0547.v1

41. Landau L. On the violation of Bell's inequality in quantum theory. Phys Lett A. (1987) 120:54-6. doi: 10.1016/0375-9601(87)90075-2

42. Cabello A. Generalized Bell nonlocality and Kochen-Specker contextuality are equivalent in quantum theory. arXiv. (2019) 1904. 05306v1.

43. Nieuwenhuizen $T$. Is the contextuality loophole fatal for the derivation of the Bell inequalities. Found Phys. (2011) 41:580-91. doi: 10.1007/s10701-010-9461-z

44. Kupczynski M. Quantum mechanics and modeling of physical reality. Phys Sci. (2018) 93:123001. doi: 10.1088/1402-4896/aae212

45. Khrennikov A. Ubiquitous Quantum Structure. New York, NY: Springer (2010). doi: 10.1007/978-3-642-05101-2

46. Asano M, Basieva I, Khrennikov A, Ohya M, Yamato I. A general quantum information model for the contextual dependent systems breaking the classical probability law. arXiv. (2011) 1105.4769v1.

47. Khrennikov A. Probability and Randomness: Quantum Versus Classical. London: Imperial College Press (2017).

48. Asano M, Basieva I, Khrennikov A, Ohya M, Yamato I. Non-Kolmogorovian approach to the context-dependent systems breaking the classical probability law. Found Phys. (2013) 43:895-911. doi: 10.1007/s10701-013-9725-5

49. Khrennikov A. Vaxjo interpretation of wave function. 2012. arXiv. (2012) 1210. 2390v1. doi: 10.1063/1.4773136

50. Busemeyer, J Bruza PD. Quantum Cognition and Decision. Cambridge: Cambridge University Press (2012). doi: 10.1017/CBO9780511997716

51. Haven E, Khrennikov A. Quantum Social Science. Cambridge: Cambridge University Press (2014).

52. Khrennikov A, Alodjants A. Classical (local and contextual) probability model for Bohm-Bell type experiments: no signaling as independence of random variables. Entropy. (2019) 21:157. doi: 10.3390/e21020157

53. Khrennikov A. Violation of Bell's inequality and postulate on simultaneous measurements of compatible observables. arXiv. (2011) 1102. 4 $743 v 1$.

54. Dzhafarov E, Kujala J, Cervantes V. Contextuality by default: a brief overview of ideas, concepts, and terminology. In: Atmanspacher H, Filk, T, Pothos, 
editors. Lecture Notes in Computer Science. New York, NY: Springer (2016). p. 12-23. doi: 10.1007/978-3-319-28675-4_2

55. Dzhafarov E, Kon M. On universality of classical probability with contextually labeled random variables. J Math Psych. (2018) 85:1724. doi: 10.1016/j.jmp.2018.06.001

56. Dzhafarov E, Kujala J. Context-content systems of random variables: the contextuality-by-default theory. J Math Psych. (2016) 74:11-33. doi: 10.1016/j.jmp.2016.04.010

57. Dzhafarov E, Zhang R, Kujala J. Is there contextuality in behavioral and social systems? Phil Trans Royal Soc A. (2015) 374:20150099. doi: 10.1098/rsta.2015.0099

58. Cervantes V, Dzhafaarov E. Snow queen is evil and beautiful: experimental evidence for probabilistic contextuality in human choices. Decision. (2018) 5:193-204. doi: 10.1037/dec0000095

59. Cervantes V, Dzhafarov E. True contextuality in a psychophysical experiment. J Math Psych. (2019) 91:119-27. doi: 10.1016/j.jmp.2019.04.006

60. Dzhafarov E; Kujala J. Contextuality analysis of the double slit experiment (with a glimpse into three slits). Entropy. (2018) 20:278. doi: 10.3390/e20040278

61. von Mises R. Probability, Statistics and Truth. New York, NY: Dover Publications (1981).

62. Pitowski, I. The Logic of Fundamental Processes: Nonmeasurable Sets and Quantum Mechanics. Ph.D. dissertation, University of Western Ontario, London, Ontario (1983).

63. Pitowski I. Resolution of the einstein-podolsky-rosen and bell paradoxes. Phys Rev Lett. (1982) 48:1299-302. doi: 10.1103/PhysRevLett.48.1299

64. Pitowski I. Deterministic model of spin and statistics. Phys Rev D. (1983) 27:2316-26. doi: 10.1103/PhysRevD.27.2316

65. Gudder S. Reality, locality, and probability. Found Phys. (1984) 14:9971010. doi: 10.1007/BF01889250

66. Gudder S. On hidden variable theories. J Math Phys. (1970) 11:4316. doi: 10.1063/1.1665156

67. Ballentine LE. Probability theory in quantum mechanics. Am J Phys. (1986) 54:883-9. doi: 10.1119/1.14783

68. Durdevic M. Contextual extensions of $C^{*}$ algebras and hidden variable theories. J Phys A Math Gen. (1990) 24:5495. doi: 10.1088/0305-4470/24/3/013

69. Durdevic M. Quantum field theory and local contextual extensions. J Phys A Math Gen. (1992) 25:665-77. doi: 10.1088/0305-4470/25/3/023

70. Griffiths R. Nonlocality claims are inconsistent with Hilbert space quantum mechanics. arXiv. (2020) 1901. 07050v3. doi: 10.1103/PhysRevA.101.022117

71. Allen J-M, Barrett J, Horsman D, Lee C, Spekkens R. Quantum common causes and quantum causal models. Phys Rev X. (2017) 7:031021. doi: 10.1103/PhysRevX.7.031021

72. Palmer T. A local deterministic model of quantum spin measurement. Proc Math Phys Sci. (1995) 451:585-608. doi: 10.1098/rspa.1995.0145

73. Laughlin R. A Different Universe: Reinventing Physics From the Bottom Down. New York, NY: Perseus Books (2005).

74. Pusey M, Barrett J, Rudolph T. On the reality of the quantum state. Nat Phys. (2012) 8:475-8. doi: 10.1038/nphys2309

75. Minev SO, Mundhada S, Shankar P, Reinhold R, Gutierrez-Jáuregui RJ, Schoelkopf, et al. To catch and reverse a quantum jump mid-flight. Nature. (2019) 570:200. doi: 10.1038/s41586-019-1287-z

76. Pokorny F, Zhang C, Higgins G, Cabello A, Klienmann M, Hennrich M. Tracking the dynamics of an ideal quantum measurement. Phys Rev Lett. (2020) 124:080401. doi: 10.1103/PhysRevLett.124.080401

77. Kocsis S, Braverman B, Ravets S, Stevens MJ, Mirin RP, Shalm LK, et al. Observing the average trajectories of single photons in a twoslit interferometer. Science. (2011) 332:1170-3. doi: 10.1126/science.12 02218

78. Lundeen J, Sutherland B, Patell A, Stewart C, Bamber C. Direct measurement of the quantum wavefunction. Nat Lett. (2011) 474:18891. doi: 10.1038/nature10120

79. Gariepy G, Krstajic N, Henderson R, Li C, Thomson R, Buller G. Heshmat $\mathrm{B}$, et al. Single-photon sensitive light-in-flight imaging. Nat Comm. (2015) 6:6021. doi: 10.1038/ncomms7408

80. Migdall A, Polyakov S, Fan J, Bienfang J (Editors.). Single-Photon Generation and Detection. New York, NY: Academic Press (2013).
81. Rauch H, Werner S. Neutron Interferometry: Lessons in Experimental Quantum Mechanics. Oxford: Oxford Science Publications (2000).

82. Weinberg S. Lectures on Quantum Mechanics. Cambridge: Cambridge University Press (2013).

83. Allahverdyan A, Balian R, Nieuwenhuizen T. Understanding quantum measurement from the solution of dynamical models. Phys Rep. (2013) 525:1-66. doi: 10.1016/j.physrep.2012.11.001

84. Allahverdyan A, Balian R, Nieuwenhuizen T. A sub-ensemble theory of ideal quantum measurement processes. Ann Phys. (2017) 376:32452. doi: 10.1016/j.aop.2016.11.001

85. Norsen T. Against "realism". Found Phys. (2007) 78:31140. doi: 10.1007/s10701-007-9104-1

86. Rosen R. Some epistemological issues in physics and biology. In: Hiley BJ, Peat FD, editors. Quantum Implications: Essays in Honour of David Bohm. London: Routledge. (1991) p. 314-27.

87. Sulis W. Naturally occurring computational systems. World Futures. (1993) 39:225-41. doi: 10.1080/02604027.1994.9972406

88. Sulis W. Causality in naturally occurring computational systems. World Futures. (1995) 44:129-48. doi: 10.1080/02604027.1995.9972538

89. Whitehead AN. Process and Reality. New York, NY: The Free Press (1978).

90. Sulis W. An information ontology for the process algebra model of non-relativistic quantum mechanics. Entropy. (2020) 22:136. doi: 10.3390/e22020136

91. Eastman TE, Keeton H (Editors.). Physics and Whitehead: Quantum, Process and Experience. Albany, NY: SUNY Press (2004).

92. Finkelstein, D. Quantum Relativity: A Synthesis of the Ideas of Einstein and Heisenberg. New York, NY: Springer (1997).

93. Hiley BJ. Process, distinction, groupoids and Clifford algebras: an alternative view of the quantum formalism. In: Coecke B, editor. New Structures for Physics. Berlin: Springer-Verlag (2001). p. 70552. doi: 10.1007/978-3-642-12821-9_12

94. Noyes HP. Bit-String Physics: A Finite and Discrete Approach to Natural Philosophy. Singapore: World Scientific (2001). doi: 10.1142/4692

95. Selesnick SA. Quanta, Logic and Spacetime: Variations on Finkelstein's Quantum Relativity. Singapore: World Scientific (1998). doi: 10.1142/3586

96. Bastin T, Kilmister CW. Combinatorial Physics. Singapore: World Scientific (1995). doi: 10.1142/2703

97. Cahill RT. Process Physics: From Information Theory to Quantum Space and Matter. New York, NY: Nova Science Publishers (2005).

98. Trofimova I. Functional constructivism: in search of formal descriptors. Nonlin Dynam Psychol Life Sci. (2017) 21:441-74. [Epub ahead of print].

99. Trofimova, I. Phenomena of Functional Differentiation and Fractal Functionality. Complex Systems Theory and Applications. WIT Press. Southhampton (2016). doi: 10.2495/DNE-V11-N4-508-521

100. Sulis W. Archetypal dynamics and emergence. In: Nation J, Trofimova I, Rand J, Sulis W, editors. Formal Descriptions of Developing Systems. Dordrecht: Kluwer Press (2002) 185-228.

101. Dewey J. Does reality possess a practical character. In: Thorndike E, editor. Essays, Philosophical and Psychological, in Honor of William JAMES, Professor in Harvard University, by his Colleagues at Columbia Univeristy. New York, NY: Longmansm, Green and Co (1908). p. 53-80.

102. Mott NF. The wave mechanics of $\alpha$-ray tracks. Proc Royal Soc London A. (1929) 126:79-84. doi: 10.1098/rspa.1929.0205

103. Reichenbach H. The Direction of Time. Reichenbach M, editors. Berkeley: University of California Press (1991).

104. Gisin N. Quantum correlation in Newtonian space and time: arbitrarily fast communication or nonlocality. arXiv. (2013) 1210. 7308v2

105. Wendel G, Martinez L, Bojowald M. Physical implication of a fundamental period of time. Phys Rev Lett. (2020) 124:241301. doi: 10.1103/PhysRevLett.124.241301

106. Wigner, E. Symmetries and Reflections: Scientific Essays. Bloomington: Indiana University Press (1967). p. 52.

107. Unger RM, Smolin, L. The Singular Universe and the Reality of Time. Cambridge: Cambridge University Press (2015). doi: 10.1017/CBO9781139696487

108. Elitzur, A. Quantum phenomena within a new theory of time. In: Elitzur A, Dolev S, Kolenda, N, editors. Quo Vadis Quantum Mechanics? New York, NY: Springer (2005). p. 325-50. doi: 10.1007/b137897 
109. Gisin N. Time really passes, science can't deny that. In: Renner R, Stupar S, editors. Time in Physics. Cham: Birkhäuser (2017). P. 1-15. doi: 10.1007/978-3-319-68655-4_1

110. Sorkin R. Relativity does not imply that the future already exists: a counterexample. In: Petkov V, editor. Relativity and the Dimensionality of the World. New York, NY: Springer (2007) 153-61. doi: 10.1007/978-1-4020-6318-3_9

111. Nelson, E. Derivation of the Schrödinger equation from Newtonian mechanics. Phys Rev. (1966) 150:1079-85. doi: 10.1103/PhysRev.150.1079

112. Adler, S. Quantum Theory as an Emergent Phenomenon. Cambridge: Cambridge University Press (2004). doi: 10.1017/CBO9780511535277

113. Levin M, Wen X.-G. Colloquium: photons and electrons as emergent phenomena. Rev Mod Phys. (2005) 77:8719. doi: 10.1103/RevModPhys.77.871

114. Damgaard FH, Huffel H. Stochastic Quantization. Singapore: World Scientific (1988). doi: 10.1142/0375

115. Bars I. Standard model of particles and forces in the framework of two time physics. Phys Rev D. (2006) 74:081095. doi: 10.1103/PhysRevD.74.085019

116. Bombelli L, Lee J, Meyer D, Sorkin R. Space-time as a causal set. Phys Rev Lett. (1987) 59:521-4. doi: 10.1103/PhysRevLett.59.521

117. Lee TD. Can time be a discrete dynamical variable? Phys Lett B. (1983) 122:217-20. doi: 10.1016/0370-2693(83)90687-1

118. Kempf A. Spacetime could be simultaneously continuous and discrete in the same way that information can. arXiv. (2010) 10104354.v1. doi: 10.1088/1367-2630/12/11/115001

119. 't Hooft G. The Cellular Automaton Interpretation of Quantum Mechanics. Berlin: Springer (2014).

120. Elze, H. Quantum models as classical cellular automata. arXiv. (2017) 1701.02252v1. doi: 10.1088/1742-6596/845/1/012022

121. Zayed AI. Advances in Shannon's Sampling Theory. Boca Raton, FL: CRC Press (1993).

122. Landau, H. Necessary density conditions for sampling and interpolation of certain entire functions. Acta Mathematica. (1967) 117:37-52. doi: 10.1007/BF02395039

123. Bisseling RH, Kosloff R. Multidimensional interpolation and differentiation based on an accelerated sinc interpolation procedure. Comp Phys Comm. (1986) 39:313-32. doi: 10.1016/0010-4655(86)90093-7
124. Markopoulou, F. The internal description of a causal set: what the universe looks like from the inside. Commun Math Phys. (2000) 211:55983.41. doi: 10.1007/s002200050826

125. Borchers HJ, Sen RN. Mathematical Implications of Einstein-Weyl Causality. New York, NY: Springer (2006). doi: 10.1007/3-540-37681-X

126. Maymon S, Oppenheim AV. Sinc interpolation of nonuniform samples. IEEE Trans Sig Processes. (2011) 59:4745-58. doi: 10.1109/TSP.2011.21 60054

127. Noether E. Invariante Variationsprobleme. Nachrichten von der Gesellschaft der Wissenschaften zu Göttingen. Mathematisch-Physikalische Klasse. (1918). 28:235-57.

128. Nagasawa M. Schrödinger Equations and Diffusion Theory. New York, NY: Birkhauser (1993). doi: 10.1007/978-3-03480560-5

129. Feynman R; Hibbs A. Quantum Mechanics and Path Integrals. New York, NY: Dover (2010).

130. Trofimova I. Sociability, diversity and compatibility in developing systems: EVS approach. In: Nation J, Trofimova I, Rand J, Sulis W, editors. Formal Descriptions of Developing Systems Kluwer: Amsterdam. (2002). p. 23148. doi: 10.1007/978-94-010-0064-2_13

131. Conway JH. On Numbers and Games. Natick, NH: A.K. Peters (2001). doi: 10.1201/9781439864159

132. Hodges W. Building Models by Games. New York, NY: Dover Publications (2006).

133. Hirsch R, Hodkinson I. Relation Algebras by Games. New York, NY: Elsevier (2002).

Conflict of Interest: The author declares that the research was conducted in the absence of any commercial or financial relationships that could be construed as a potential conflict of interest.

Copyright (C) 2020 Sulis. This is an open-access article distributed under the terms of the Creative Commons Attribution License (CC BY). The use, distribution or reproduction in other forums is permitted, provided the original author $(s)$ and the copyright owner(s) are credited and that the original publication in this journal is cited, in accordance with accepted academic practice. No use, distribution or reproduction is permitted which does not comply with these terms. 